UM-P-92/28

OZ-92/08

\title{
Systematic Study of Fermion Masses and Mixing Angles in Horizontal SU(2) Gauge Theory
}

\author{
D. S. Shaw and R. R. Volkas \\ Research Centre for High Energy Physics, School of Physics, \\ University of Melbourne, Parkville 3052, Australia
}

\begin{abstract}
Despite its great success in explaining the basic interactions of nature, the standard model suffers from an inability to explain the observed masses of the fundamental particles and the weak mixing angles between them. We shall survey a set of possible extensions to the standard model, employing an $\mathrm{SU}(2)$ "horizontal" gauge symmetry between the particle generations, to see what light they can shed on this problem.
\end{abstract}

\section{INTRODUCTION}

One of the most important unresolved problems in particle physics is the origin of the fermion mass spectrum and the mixing angles between the quark fields. One avenue of investigation into this problem is that of horizontal symmetry. This idea proposes a new symmetry to exist between the different generations of particles. Various symmetries have been investigated in the literature, both discrete [1] and continuous. Possible continuous

symmetries to look at are $U(1)$ [ [6], $S U(2)$ [15] and $S U(3)$ [16] symmetries. While there has been considerable work on $S U(2)$ horizontal symmetry models, such investigations have been based on only a few possible sets of fermion and Higgs boson assignments. 
In this paper we shall survey all the possible particle assignments under $S U(2)$ looking at the predictions made by each model for the tree level mass spectrum and the tree level Kobayashi-Maskawa (KM) matrix. The gauge group is thus taken to be $S U(3)_{C} \otimes S U(2)_{H} \otimes$ $S U(2)_{L} \otimes U(1)_{Y}$, where $S U(3)_{C} \otimes S U(2)_{L} \otimes U(1)_{Y}$ is the standard model gauge group and $S U(2)_{H}$ is the horizontal gauge group.

Before investigating this extended model, we shall take a brief look at the qualitative features of the observed fermion mass and mixing angle spectrum, to gain an idea of the sort of results we wish to see coming from the models incorporating horizontal symmetry.

Table — displays the masses of the fundamental charged fermions (neutrinos are taken to be massless in the first part of this report - bounds on the neutrino masses will be given in Sec. [V]). The first and most obvious thing to note about the mass spectrum is the progressive increase in mass with each generation. One way for this to come about, and the view that is taken in this paper, is for the higher generation masses to result from first-order, or tree-level, terms in the lagrangian. In this paper, these tree-level interactions are taken to be Yukawa couplings between fermion fields and Higgs multiplets. The horizontal symmetry is spontaneously broken, with neutral Higgs components acquiring non-zero vacuum expectation values (VEVs), and through the tree-level Yukawa interactions with the particle fields, this generates the larger masses and mixing angles. The earlier generation masses are then presumed to result from radiative corrections at the 1-loop level and higher. To generate these radiative corrections will require further extensions to the horizontal model. What we hope to see in the models investigated in this report then, are tree-level mass spectra that show masses for the heavier fermions and larger mixing angles, while leaving the lighter fermion masses and smaller mixing angles to be generated through higher order interactions.

Also notable about the mass spectrum is an approximate hierarchy between the fermion sectors, with each charge $\frac{2}{3}$ quark (the "up" quarks) being heavier in general than the charge $-\frac{1}{3}$ quark (the "down" quark) of the same generation, which in turn is heavier than the charged lepton of that generation. The exception to this general trend is the up quark, whose mass is a little lighter than the down quark mass. Hence, we might expect to see 
tree-level masses for the "up" quarks only, or maybe the "down" quarks as well, while the charged leptons (at least the lighter ones) would probably only gain masses through higher-order corrections, which would be expected to be naturally smaller.

There is also the matter of the mixing angles between the two quark sectors. Eq. (11) displays the experimentally determined values for the entries in the Kobayashi-Maskawa (KM) matrix [20]. To a first approximation, the KM matrix can be taken as the identity matrix, so following the above prescription, one would hope to find this appearing at the tree level. Alternatively would be a model that also generates the Cabibbo angle at tree level, as this angle is of a comparable size to the diagonal entries. The other two mixing angles are, however, relatively small, so that while it may be feasible to generate the mixing angle between the second and third generations at tree level (although this would require some accurate cancellations between the parameters of the model to keep this value small), it would be very improbable that a model would naturally generate a tree level value for the mixing angle between the first and third generations without also generating one or both of the other angles.

$$
U_{K M}=\left(\begin{array}{ccc}
0.9747-0.9759 & 0.218-0.224 & 0.001-0.007 \\
0.218-0.224 & 0.9734-0.9752 & 0.030-0.058 \\
0.003-0.019 & 0.029-0.058 & 0.9983-0.9996
\end{array}\right)
$$

As will be seen, many of the models give quite reasonable mass spectra, but they often have trouble with these mixing angle hierarchy problems. Six models, however, do show a fair amount of promise.

The plan of the remainder of this paper is as follows: Sec. II tables the possible representations under $S U(2)_{H}$ for the various fermion fields and all possible particle assignments consistent with anomaly cancellation in the absence of right-handed neutrinos are determined. A sample analysis of one model is given. Sec. III presents an analysis of the results for the various possible Higgs multiplets in each model (while it is certainly feasible to combine various of the possible Higgs multiplets within a model, in this report the results for 
each Higgs multiplet within a given model will be looked at individually, with only occasional comments on combining multiplets). In Sec. IV], the effects of adding in right-handed neutrinos are investigated. In Sec. $\nabla$, limits on horizontal gauge boson masses and problems associated with horizontal symmetries are discussed and one method for resolving some of these problems is presented. Sec. $\nabla]$ gives a summary of important results.

\section{ANOMALY-FREE $S U(2)_{H}$ SPECTRA IN THE ABSENCE OF RIGHT-HANDED NEUTRINOS}

The number of generations of particles is taken to be three. This is in accord with data on the $\mathrm{Z}$ width from the LEP facility [21] which places the number of light neutrinos at

$$
N_{\nu}=3.00 \pm 0.05
$$

While this does not exclude higher generations, the neutrinos in these generations would be very massive and initially we will be considering only models with massless neutrinos (no right-handed neutrinos). Later, the possibility of including right-handed neutrinos will be looked at, although the number of generations will still be taken to be three. With three generations, each of the fermion fields $l_{\mathrm{L}}, e_{\mathrm{R}}, q_{\mathrm{L}}, u_{\mathrm{R}}$ and $d_{\mathrm{R}}$ may transform under $S U(2)_{H}$ as either a triplet, a doublet and a singlet or as three singlets. To decide which of these combinations of representations are reasonable, global and gauge anomaly cancellation is imposed. Gauge anomaly cancellation is satisfied if the $\left[\mathrm{SU}(2)_{\mathrm{H}}\right]^{2} \mathrm{U}(1)_{\mathrm{Y}}$ anomaly cancels out between the lepton and quark fields, while global anomaly cancellation requires that there be an even number of $\mathrm{SU}(2)_{\mathrm{H}}$ doublets. Tables $\mathbb{I}$ and $[\mathrm{II}$ show the values for these anomalies for the various possible ways of assigning representations to the particle types.

Comparing the values from these tables, fourteen theories satisfying gauge and global anomaly cancellation appear. They are: 


$$
\begin{array}{cc}
\mathrm{L} 1+\mathrm{Q} 1, & \mathrm{~L} 1+\mathrm{Q} 8, \\
\mathrm{~L} 2+\mathrm{Q} 6, & \mathrm{~L} 3+\mathrm{Q} 4, \\
\mathrm{~L} 5+\mathrm{Q} 12, & \mathrm{~L} 5+\mathrm{Q} 13, \\
\mathrm{~L} 6+\mathrm{Q} 10, & \mathrm{~L} 6+\mathrm{Q} 11, \\
\mathrm{~L} 7+\mathrm{Q} 18, & \mathrm{~L} 8+\mathrm{Q} 16, \\
\mathrm{~L} 1+\mathrm{Q} 21, & \mathrm{~L} 9+\mathrm{Q} 1, \\
\mathrm{~L} 9+\mathrm{Q} 8, & \mathrm{~L} 9+\mathrm{Q} 21 .
\end{array}
$$

The last of these models is just the standard model, and therefore of little interest to the present endeavour. For each of the other models, the Yukawa couplings between the fermions and Higgs particles will be found, and the possible Higgs multiplets for that model determined. From there, the pattern of masses and mixing angles will be found. For the sake of brevity, only one model is worked out in any detail here, with the next section providing a table of results, and a discussion of the features of the models.

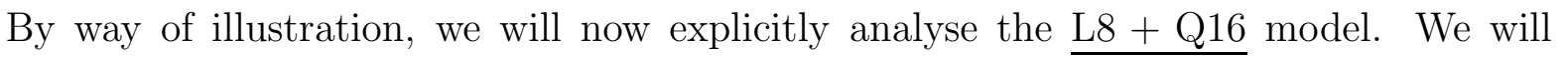
first determine which Higgs multiplets can couple to the quark and lepton bilinears through Yukawa coupling terms. We will then take each possible Higgs multiplet in isolation, and determine what fermion mass and mixing angle spectrum results after spontaneous symmetry breaking. The fermion assignments under $\mathrm{SU}(3)_{\mathrm{C}} \otimes \mathrm{SU}(2)_{\mathrm{H}} \otimes \mathrm{SU}(2)_{\mathrm{L}} \otimes \mathrm{U}(1)_{\mathrm{Y}}$ for this model are found to be:

$$
\begin{array}{lll}
l_{i L} \sim(1,1,2)(-1), i=1,2,3, & & \\
e_{R} \sim(1,2,1)(-2), & & e_{3 R} \sim(1,1,1)(-2), \\
q_{L} \sim(3,2,2)\left(\frac{1}{3}\right), & & q_{3 L} \sim(3,1,2)\left(\frac{1}{3}\right), \\
u_{R} \sim(3,2,1)\left(\frac{4}{3}\right), & & u_{3 R} \sim(3,1,1)\left(\frac{4}{3}\right), \\
d_{i R} & \sim(3,1,1)\left(-\frac{2}{3}\right), i=1,2,3 . &
\end{array}
$$

This gives the Higgs-fermion interactions

$$
\begin{aligned}
\mathcal{L}_{\text {Yukawa }}^{\mathrm{H}-\mathrm{f}}= & \lambda_{1 i} \bar{l}_{i L} e_{R} \chi^{C}+\lambda_{2 i} \bar{l}_{i L} e_{3 R} \phi^{C}+\lambda_{3} \bar{q}_{L} u_{R} \phi \\
& +\lambda_{4} \bar{q}_{L} u_{R} \Delta+\lambda_{5} \bar{q}_{L} u_{3 R} \chi+\lambda_{6 i} \bar{q}_{L} d_{i R} \chi^{C}
\end{aligned}
$$




$$
+\lambda_{7} \bar{q}_{3 L} u_{R} \chi+\lambda_{8} \bar{q}_{3 L} u_{3 R} \phi+\lambda_{9 i} \bar{q}_{3 L} d_{i R} \phi^{C}+\text { H.c. },
$$

where the Higgs multiplets are

$$
\begin{aligned}
& \phi \sim(1,1,2)(-1), \\
& \chi \sim(1,2,2)(-1), \\
& \Delta \sim(1,3,2)(-1),
\end{aligned}
$$

and $\phi^{C}$ and $\chi^{C}$ are the charge conjugates of $\phi$ and $\chi$ respectively $-\phi^{C} \equiv i \tau_{2} \phi^{*}$ where $\tau_{2}$ is the usual Pauli matrix operating in $S U(2)_{L}$ space and $\chi^{C}$ results from similar transformations in both $S U(2)_{L}$ space and $S U(2)_{H}$ space.

We look at each of these three Higgs possibilities in isolation. First, consider the terms involving $\phi$. This Higgs multiplet has one neutral component, which gains a vacuum expectation value (VEV), spontaneously breaking the gauge symmetry. Setting $\left\langle\phi^{0}\right\rangle=v$ gives the mass terms

$$
\mathcal{L}_{\text {Mass }}^{\text {fermion }}=\bar{U}_{L}^{\prime} M^{U} U_{R}^{\prime}+\bar{D}_{L}^{\prime} M^{D} D_{R}^{\prime}+\bar{E}_{L}^{\prime} M^{E} E_{R}^{\prime}+\text { H.c. }
$$

where $U^{\prime}=\left(u^{\prime}, c^{\prime}, t^{\prime}\right)^{T}, D^{\prime}=\left(d^{\prime}, s^{\prime}, b^{\prime}\right)^{T}, E^{\prime}=\left(e^{\prime}, \mu^{\prime}, \tau^{\prime}\right)^{T}$ (primes are used to denote that these are weak eigenstates, not mass eigenstates). Also,

$$
\begin{gathered}
M^{U}=\left(\begin{array}{ccc}
\lambda_{3} v & 0 & 0 \\
0 & \lambda_{3} v & 0 \\
0 & 0 & \lambda_{8} v
\end{array}\right)=D^{U}, \\
M^{D}=\left(\begin{array}{ccc}
0 & 0 & 0 \\
0 & 0 & 0 \\
\lambda_{91} v & \lambda_{92} v & \lambda_{93} v
\end{array}\right), \\
M^{E}=\left(\begin{array}{ccc}
0 & 0 & \lambda_{21} v \\
0 & 0 & \lambda_{22} v \\
0 & 0 & \lambda_{23} v
\end{array}\right),
\end{gathered}
$$


where $M^{U}, M^{D}, M^{E}$ are the (undiagonalised) mass matrices for the charge $\frac{2}{3}$ quarks, charge $-\frac{1}{3}$ quarks and the charge -1 leptons respectively, and $D^{U}, D^{D}, D^{E}$ are the corresponding diagonalised (mass eigenstate) matrices. The mass eigenstate fields are of the form

$$
\begin{gathered}
U_{L}^{\prime}=A_{L} U_{L}, U_{R}^{\prime}=A_{R} U_{R}, D_{L}^{\prime}=B_{L} D_{L}, \\
D_{R}^{\prime}=B_{R} D_{R}, E_{L}^{\prime}=C_{L} E_{L}, E_{R}^{\prime}=C_{R} E_{R},
\end{gathered}
$$

where $A_{L, R}, B_{L, R}, C_{L, R}$ are unitary matrices, and

$$
A_{L}^{\dagger} M^{U} A_{R}=D^{U}, B_{L}^{\dagger} M^{D} B_{R}=D^{D}, C_{L}^{\dagger} M^{E} C_{R}=D^{E} .
$$

Clearly $A_{L}=A_{R}=I_{3 \times 3}$. It turns out that the simplest choice for $B_{L}$ is $I_{3 \times 3}$ as well. Thus the KM matrix, given by $U_{K M}=A_{L}^{\dagger} B_{L}$, is simply the identity matrix at tree level. $D^{D}$ and $D^{E}$ are found to be:

$$
\begin{aligned}
& D^{D}=\left(\lambda_{91}^{2}+\lambda_{92}^{2}+\lambda_{93}^{2}\right)^{\frac{1}{2}} v\left(\begin{array}{ccc}
0 & 0 & 0 \\
0 & 0 & 0 \\
0 & 0 & 1
\end{array}\right), \\
& D^{E}=\left(\lambda_{21}^{2}+\lambda_{22}^{2}+\lambda_{23}^{2}\right)^{\frac{1}{2}} v\left(\begin{array}{ccc}
0 & 0 & 0 \\
0 & 0 & 0 \\
0 & 0 & 1
\end{array}\right) .
\end{aligned}
$$

So the tree level results for this model are:

Massive : $\mathrm{u}, \mathrm{c}, \mathrm{t}$ and $\mathrm{b}$ quarks and the lepton $\tau$.

Massless : $\mathrm{d}$ and s quarks and the leptons e and $\mu$.

KM matrix : $U_{K M}=I_{3 \times 3}$.

These tree-level results are quite good, with the exception of the up-quark sector. Here we have either

$$
m_{u}=m_{c} \quad \text { or } \quad m_{c}=m_{t} .
$$


Observationally, $m_{t} \gg m_{c} \gg m_{u}$. In order to satisfy this, one must generate radiative corrections of similar magnitude to the tree level results. If, for example, we take $m_{c}=m_{u}$ at tree level, we need to generate radiative corrections of the order of the (tree-level) charm quark mass, splitting the masses of the up and charm quarks, and balanced so as to cancel the up quark mass almost exactly (in comparison with the magnitude of the charm quark mass). While this is not impossible, it seems unlikely that such precise balancing would occur naturally.

We now move on to the next Higgs multiplet, $\chi \sim \underline{2}$ (under $\mathrm{SU}(2)_{H}$ ), which transforms according to

$$
\chi \rightarrow U_{L} \chi U_{H}^{T}
$$

where $U_{L}$ represents a (unitary) rotation in $S U(2)_{L}$ space, and $U_{H}$ is a (unitary) rotation in the horizontal space. In matrix notation,

$$
\chi=\left(\begin{array}{ll}
\chi_{1}^{0} & \chi_{2}^{0} \\
\chi_{1}^{-} & \chi_{2}^{-}
\end{array}\right) .
$$

Both neutral components will gain VEVs when the horizontal symmetry is broken: $\left\langle\chi_{1}^{0}\right\rangle=w_{1},\left\langle\chi_{2}^{0}\right\rangle=w_{2}$. This leads to

$$
\begin{gathered}
M^{U}=\left(\begin{array}{ccc}
0 & \lambda_{5} w_{2} & 0 \\
-\lambda_{7} w_{1} & 0 & \lambda_{7} w_{2} \\
0 & \lambda_{5} w_{1} & 0
\end{array}\right), \\
M^{D}=\left(\begin{array}{ccc}
-\lambda_{61} w_{1} & -\lambda_{62} w_{1} & -\lambda_{63} w_{1} \\
0 & 0 & 0 \\
\lambda_{61} w_{2} & \lambda_{62} w_{2} & \lambda_{63} w_{2}
\end{array}\right), \\
M^{E}=\left(\begin{array}{ccc}
\lambda_{11} w_{2} & 0 & \lambda_{11} w_{1} \\
\lambda_{12} w_{2} & 0 & \lambda_{12} w_{1} \\
\lambda_{13} w_{2} & 0 & \lambda_{13} w_{1}
\end{array}\right) .
\end{gathered}
$$


Diagonalising these matrices gives

$$
\begin{gathered}
D^{U}=\left(w_{1}^{2}+w_{2}^{2}\right)^{-\frac{1}{2}}\left(\begin{array}{ccc}
0 & 0 & 0 \\
0 & \lambda_{7} & 0 \\
0 & 0 & \lambda_{5}
\end{array}\right), \\
D^{D}=\left[\left(\lambda_{61}^{2}+\lambda_{62}^{2}+\lambda_{63}^{2}\right)\left(w_{1}^{2}+w_{2}^{2}\right)\right]^{\frac{1}{2}}\left(\begin{array}{ccc}
0 & 0 & 0 \\
0 & 0 & 0 \\
0 & 0 & 1
\end{array}\right), \\
D^{E}=\left[\left(\lambda_{11}^{2}+\lambda_{12}^{2}+\lambda_{13}^{2}\right)\left(w_{1}^{2}+w_{2}^{2}\right)\right]^{\frac{1}{2}}\left(\begin{array}{ccc}
0 & 0 & 0 \\
0 & 0 & 0 \\
0 & 0 & 1
\end{array}\right),
\end{gathered}
$$

along with

$$
\begin{gathered}
A_{L}=\left(w_{1}^{2}+w_{2}^{2}\right)^{-\frac{1}{2}}\left(\begin{array}{ccc}
w_{1} & 0 & w_{2} \\
0 & \left(w_{1}^{2}+w_{2}^{2}\right)^{\frac{1}{2}} & 0 \\
-w_{2} & 0 & w_{1}
\end{array}\right), \\
B_{L}=\left(w_{1}^{2}+w_{2}^{2}\right)^{-\frac{1}{2}}\left(\begin{array}{ccc}
w_{2} & 0 & -w_{1} \\
0 & \left(w_{1}^{2}+w_{2}^{2}\right)^{\frac{1}{2}} & 0 \\
w_{1} & 0 & w_{2}
\end{array}\right) .
\end{gathered}
$$

Hence the KM matrix is

$$
U_{K M}=\left(\begin{array}{ccc}
0 & 0 & -1 \\
0 & 1 & 0 \\
1 & 0 & 0
\end{array}\right) .
$$

In such cases, a second $\chi$ Higgs multiplet may be added (see ref. [14]), having the effect of altering $U_{K M}$ to 


$$
U_{K M}=\left(\begin{array}{ccc}
\cos \theta & 0 & \sin \theta \\
0 & 1 & 0 \\
-\sin \theta & 0 & \cos \theta
\end{array}\right) .
$$

It would be quite impressive if the angle $\theta$ could be identified with the Cabibbo angle, however the location of the non-zero entries in the diagonalised matrices $D^{U}$ and $D^{D}$ precludes such an interpretation. The bottom-right entry in $D^{D}$ is taken to be the bottom quark mass, implying that $\theta$ represents mixing between the third and first generations (where $m_{t}$ is taken to be $\lambda_{5}$ and $m_{c}$ is taken to be $\lambda_{7}$, that is, the bases between which the KM matrix in Eq. (28) operates are the standard bases $(\mathrm{u}, \mathrm{c}, \mathrm{t})$ and $(\mathrm{d}, \mathrm{s}, \mathrm{b})$ - other assignments are possible but lead to KM matrices with zero diagonal entries). Since experimentally the KM matrix entries $K_{13}$ and $K_{31}$ are found to be very small $(\sim 0.004)$, this is not a good result. It is possible that the parameters of the model could be made to cancel so exactly as to yield such a small quantity for $\theta$ and also generate the much larger Cabibbo angle through radiative corrections, but the aim is to find models in which this happens naturally, rather than requiring the parameters to be carefully set "by hand."

Hence, while the mass spectrum for this model is excellent, providing tree level masses for t,c,b and $\tau$ (in line with the observed relations $m_{t}>m_{c} \simeq m_{b} \simeq m_{\tau}>$ all other masses), the model suffers from a severe hierarchy problem in the KM matrix.

A lesser problem, but one that crops up frequently, is the hierarchy between those masses that are generated at tree level. In this case, the same term in the lagrangian has generated (generally) different masses for the top and the charmed quark, but a large difference between the values of the coefficients $\lambda_{5}$ and $\lambda_{7}$ would still be required to explain why the observed split between the masses of these two particles is as great as it is (approximately two orders of magnitude).

The last Higgs multiplet for the $\underline{L_{8}+Q_{16}}$ model is the $\Delta \sim \underline{3}$ multiplet. This is a symmetric tensor in $S U(2)_{H}$ space, $\Delta_{\alpha}^{i j}$, where $\alpha=1,2$ indexes the weak isospin of the components and $i, j=1,2$ are $S U(2)_{H}$ indices. The $\Delta$ Higgs transforms according to 


$$
\Delta_{\alpha}^{i j} \rightarrow \Delta_{\beta}^{k l}=\left(U_{L}\right)_{\beta}^{\alpha}\left(U_{H}\right)_{i}^{k}\left(U_{H}\right)_{j}^{l} \Delta_{\alpha}^{i j}
$$

There are three neutral components, $\Delta_{1}^{0}, \Delta_{2}^{0}$ and $\Delta_{3}^{0}$ :

$$
\Delta_{(\alpha=1)}^{i j}=\left(\begin{array}{cc}
\Delta_{1}^{0} & \frac{\Delta_{2}^{0}}{\sqrt{2}} \\
\frac{\Delta_{2}^{0}}{\sqrt{2}} & \Delta_{3}^{0}
\end{array}\right) .
$$

These neutral components acquire VEVs $\left\langle\Delta_{1}^{0}\right\rangle=x_{1},\left\langle\Delta_{2}^{0}\right\rangle=x_{2},\left\langle\Delta_{3}^{0}\right\rangle=x_{3}$. Only the "up" quarks couple to this Higgs, hence the "down" quarks and the charged leptons will remain massless at tree level. Furthermore the KM matrix becomes irrelevant at tree level as the massless "down" quark states may be freely rotated into superpositions of each other, making the KM matrix elements unphysical. In the "up" quark sector,

$$
M^{U}=\left(\begin{array}{ccc}
\frac{x_{2}}{\sqrt{2}} & -x_{1} & 0 \\
x_{3} & -\frac{x_{2}}{\sqrt{2}} & 0 \\
0 & 0 & 0
\end{array}\right),
$$

leading to two massive quarks (c and t) upon diagonalising. This easily explains the fermion

sector hierarchy, with the charge $\frac{2}{3}$ quarks having greater masses than the other fermions of their respective generations, leaving room also for the up and down quarks to have comparable masses, but it seems a little strange to require that radiative corrections give masses to the bottom quark and the $\tau$ comparable to the charm quark mass (it is possible if the radiative corrections are proportional to the top quark mass, but the hierarchy between the top and charmed quarks is unexplained).

\section{TREE LEVEL RESULTS}

Having analysed one model in detail, we now turn to table $\mathbb{D}$ which provides a summary of the results for all of the models (excepting the SM) in Eq. (3).

As can be seen from this table, in many cases the mass spectrum is found to give satisfactory results. Typically, the third generation only will receive masses, leaving the other generations to obtain their lesser masses through radiative corrections. This occurs in 
the models $\underline{L_{7}+Q_{18}}, \underline{L_{6}+Q_{11}}, \underline{L_{5}+Q_{12}}, \underline{L_{3}+Q_{4}}$ (for the $\Delta$ Higgs multiplet) and $\underline{L_{1}+Q_{1}}$ $(\Delta$ Higgs multiplet).

In other cases some or all of the second generation particles also gain tree-level masses (the $\chi$ Higgs multiplet in $\underline{L_{8}+Q_{16}}, \Delta$ in $\underline{L_{6}+Q_{10}}, \chi$ and $\zeta$ in $\underline{L_{3}+Q_{4}}, \chi$ and $\Delta$ in $\underline{L_{2}+Q_{6}}$, and $\Delta$ in $\underline{L_{1}+Q_{8}}$ ), although a couple of these models yield strange relations, not existent in the observed spectrum, which seem unlikely to be resolved naturally by the radiative corrections (an example of this is the $\chi \sim \underline{2}$ Higgs multiplet from the model $\underline{L_{3}+Q_{4}}$ in which the second generation masses are found to be related to the third generation masses by the equation $m_{2}=\frac{m_{3}}{\sqrt{2}}$ - a relation which needs to be strongly broken by higher-order effects in order to match the observed difference in masses between these two generations. The $\chi$ Higgs multiplet from the model $\underline{L_{2}+Q_{6}}$ also suffers from this defect).

A further problem with such models is that, while the one Yukawa coupling term may generate different masses for two or three of the fermions in a given sector (for instance, to the charm and top quarks), it is hard to accept that this will naturally explain the order-of-magnitudes splitting between the masses of particles of different generations.

Another pattern occasionally emerging is for just one or two sectors (for example, only the "up" quarks) to be given masses. If the sectors that receive masses are the heavier "up" quarks - possibly along with the "down" quarks — then this is quite feasible, especially if radiative corrections then couple together particles within each generation, so that the bottom quark and the tauon receive masses by interactions with the top quark and so on for the other generations. Such a pattern agrees with the observed hierarchy between the sectors, however the observed relation $m_{u} \lesssim m_{d}$ may prove troublesome in such a scheme. Models that follow this pattern are $\underline{L_{8}+Q_{16}}\left(\Delta\right.$ Higgs multiplet), $\underline{L_{6}+Q_{10}}(\phi$ and $\psi$ ), $\underline{L_{5}+Q_{13}}(\phi), \underline{L_{2}+Q_{6}}(\phi), \underline{L_{1}+Q_{8}}(\chi), \underline{L_{9}+Q_{8}}(\chi$ and $\Delta)$ and $\underline{L_{9}+Q_{1}}$ (for the $\Delta$ and $\psi$ Higgs multiplets).

There are also a few models generating masses for all the particles. Most, such as the $\phi \sim \underline{1}$ Higgs multiplet possibility from the model $\underline{L_{1}+Q_{8}}$, that have equal masses (here, the 
leptons all have the same mass while two charge $\frac{2}{3}$ quarks share one mass and two charge $-\frac{1}{3}$ quarks share another) seem a little unlikely (other models in this category are $\underline{L_{1}+Q_{1}}$, $\underline{L_{9}+Q_{8}}, \underline{L_{9}+Q_{1}}$ and $\underline{L_{1}+Q_{21}}$ - looking at the $\phi$ Higgs multiplet in each case). The one case that avoids these dubious equalities, the $\psi$ Higgs transforming as a $\underline{5}$ under $S U(2)_{H}$ in the $\underline{L_{1}+Q_{1}}$ model, may provide some reasonable relations reducing the arbitrariness of the mass spectrum and the KM matrix, but the algebra can be extremely dense and this case is of limited interest. This model has, however, received some attention from Wilczek and Zee 11] among others 12,13]. Their work focuses on a model combining the $\Delta$ and $\psi$ multiplets from $\underline{L_{1}+Q_{1}}$, in which the form of the Higgs potentials reduces the number of non-zero components in the Higgs multiplets. The result is a model that generates tree level masses for the second and third generations, along with $U_{K M}=I_{3 \times 3}$.

Although many of the models produce satisfactory results for the mass spectrum of the particles, the KM matrix is often not so good. For those cases where one of the quark sectors remains totally free of any masses at tree level, then the KM matrix entries will be unphysical (as is the case for the $\Delta$ Higgs possibility from $\underline{L_{8}+Q_{16}}$, looked at in Sec. ㅍII). Nothing further can be said about these models until the nature of the radiative corrections is determined.

An occasional model produces a KM matrix such as

$$
U_{K M}=\left(\begin{array}{ccc}
0 & z & z \\
0 & z & z \\
z & 0 & 0
\end{array}\right),
$$

where " $z$ " represents a non-zero entry. These are tabulated as "zero diagonal entries", and clearly differ from the observed form for this matrix.

Many models produced finite values for some or all of the off-diagonal entries in the KM matrix. Where the first and second generation members of one (or both) quark sector(s) have zero mass at tree level, the corresponding $2 \times 2$ sub-matrix in the KM matrix is unphysical, and these entries can be eliminated. Often this leaves a non-zero result for the angle(s) mixing the second and third generation quarks and/or the first and third generation quarks. 
One then has a hierarchy problem in explaining how these angles eventually acquire their observed values. The problem is particularly severe where the 1-3 generation mixing angle is non-zero, as this angle is measured to have a value of about .004 that of the diagonal entries, a hierarchy of the order of $1 / 200$. The $2-3$ generation mixing angle hierarchy is less severe $(\sim 1 / 20)$, and models generating this angle at tree level are feasible - though not very satisfactory.

The most hopeful models are those for which the tree-level KM matrix is simply the identity matrix, or those which generate a finite value for only the 1-2 generation mixing angle (the Cabibbo angle).

Of all the models, then, taking into account both the mass spectra and the KM matrix results, six models look most promising:

The first of these cases is the model $\underline{L_{7}+Q_{18}}$ with the $\phi \sim \underline{1}$ Higgs multiplet. The tree-level predictions for this model are:

Massive : top and bottom quarks and the lepton $\tau$.

Massless : up, down, charm and strange quarks and the leptons e and $\mu$.

KM matrix : $U_{K M}=I_{3 \times 3}$.

It is left to radiative corrections to assign smaller masses to the initially massless first and second generation particles and to provide values for the off-diagonal entries in the KM matrix. This particular model has been looked at by Babu and Mohapatra [15]. In their paper, Babu and Mohapatra obtain radiative corrections for the model by proposing the coexistence of the $\chi \sim \underline{2}$ Higgs multiplet and also adding scalar fields of the type we describe in Sec. $\square$. By assuming conservation of the tau lepton number, and assigning $L_{\tau}=-1$ to the $\chi$ multiplet, the neutral components of this multiplet are prevented from gaining VEVs, thus leaving the mass matrices unchanged at the tree level, while allowing for radiative corrections at the 1-loop level and beyond.

The second model is $\underline{L_{3}+Q_{4}}$ with the $\Delta \sim \underline{3}$ Higgs multiplet. The predictions from this 
model are identical to those of the previous model, namely tree level masses for the third generation particles (t,b and $\tau$ ) and a tree-level KM matrix equal to the identity matrix.

The next model, $\underline{L_{1}+Q_{8}}$ with the $\Delta \sim \underline{3}$ Higgs multiplet, generates masses for most of the first and second generation particles:

Massive : top, bottom, charm and strange quarks and the lepton $\tau$.

Massless : up and down quarks and the leptons e and $\mu$.

KM matrix : $U_{K M}=I_{3 \times 3}$.

This approximates very well with the observed mass spectrum, and again gives $U_{K M}=I_{3 \times 3}$ at tree level, although the origin of the hierarchy between the second and third generations is not clear from these tree-level results.

The $\underline{L_{1}+Q_{8}}$ model with the $\chi \sim \underline{2}$ Higgs multiplet proposes tree level masses for the four heaviest quarks (t,b,c and s) leaving all the leptons massless. Also, if a second $\chi$ Higgs multiplet is added in, this model generates the Cabibbo angle at tree level. This is an excellent result, except for the lack of a tree level mass for the tauon. The masslessness of the leptons could possibly be fixed by combining the $\chi$ Higgs multiplet with the $\psi \sim \underline{5}$ multiplet, which generates (distinct) masses for all three leptons. If this is done then the radiative corrections will need to be able to explain how the initially massless first generation quarks $(u$ and $d)$ receive masses generated at higher order which are of greater magnitude than the (tree-level) electron mass.

Finally, two Higgs multiplets from the $\underline{L_{9}+Q_{8}}$ model look promising. In this model, only the quark fields transform in a non-trivial manner under the horizontal symmetry, the leptons behaving just as they do in the SM. Both of the Higgs multiplets generate masses for the second and third generation quarks, leaving the leptons massless at tree level. The $\chi \sim \underline{2}$ Higgs multiplet generates a tree level Cabibbo angle, while the other Higgs multiplet, $\Delta \sim \underline{3}$ leaves the KM matrix equal to the identity matrix at tree level. Again, these results are in good agreement with the observed mass spectrum, but the masslessness of the tauon 
is a little problematical.

\section{MODELS INCORPORATING RIGHT-HANDED NEUTRINOS}

This section will look at the effect of including right-handed neutrinos (RHNs) in the particle spectrum. Since these neutrino fields are invariant under transformations of the SM gauge group (although not necessarily under the horizontal gauge group), there will be no contribution from these fields to the gauge anomaly. The contribution of the RHN fields to the global anomaly will also be zero if the neutrinos transform either as three singlets or as a triplet under $S U(2)_{H}$ ("N1" or "N3" respectively), and will be one doublet if the fields transform as a singlet and a doublet ("N2"). This leads to a total of 35 models for analysis: 


$$
\begin{array}{lc}
\mathrm{L} 1+\mathrm{Q} 1+\mathrm{N} 1, & \mathrm{~L} 1+\mathrm{Q} 1+\mathrm{N} 3, \\
\mathrm{~L} 1+\mathrm{Q} 8+\mathrm{N} 1, & \mathrm{~L} 1+\mathrm{Q} 8+\mathrm{N} 3, \\
\mathrm{~L} 2+\mathrm{Q} 5+\mathrm{N} 2, & \\
\mathrm{~L} 2+\mathrm{Q} 6+\mathrm{N} 1, & \mathrm{~L} 2+\mathrm{Q} 6+\mathrm{N} 3, \\
\mathrm{~L} 3+\mathrm{Q} 3+\mathrm{N} 2, & \\
\mathrm{~L} 3+\mathrm{Q} 4+\mathrm{N} 1, & \mathrm{~L} 3+\mathrm{Q} 4+\mathrm{N} 3, \\
\mathrm{~L} 4+\mathrm{Q} 1+\mathrm{N} 2, & \\
\mathrm{~L} 4+\mathrm{Q} 8+\mathrm{N} 2, & \\
\mathrm{~L} 5+\mathrm{Q} 12+\mathrm{N} 1, & \mathrm{~L} 5+\mathrm{Q} 12+\mathrm{N} 3, \\
\mathrm{~L} 5+\mathrm{Q} 13+\mathrm{N} 1, & \mathrm{~L} 5+\mathrm{Q} 13+\mathrm{N} 3, \\
\mathrm{~L} 6+\mathrm{Q} 10+\mathrm{N} 1, & \mathrm{~L} 6+\mathrm{Q} 10+\mathrm{N} 3, \\
\mathrm{~L} 6+\mathrm{Q} 11+\mathrm{N} 1, & \mathrm{~L} 6+\mathrm{Q} 11+\mathrm{N} 3, \\
\mathrm{~L} 7+\mathrm{Q} 18+\mathrm{N} 1, & \mathrm{~L} 7+\mathrm{Q} 18+\mathrm{N} 3, \\
\mathrm{~L} 7+\mathrm{Q} 19+\mathrm{N} 2, & \\
\mathrm{~L} 8+\mathrm{Q} 16+\mathrm{N} 1, & \mathrm{~L} 8+\mathrm{Q} 16+\mathrm{N} 3, \\
\mathrm{~L} 8+\mathrm{Q} 17+\mathrm{N} 2, & \\
\mathrm{~L} 1+\mathrm{Q} 21+\mathrm{N} 1, & \mathrm{~L} 1+\mathrm{Q} 21+\mathrm{N} 3, \\
\mathrm{~L} 4+\mathrm{Q} 21+\mathrm{N} 2, & \\
\mathrm{~L} 9+\mathrm{Q} 1+\mathrm{N} 1, & \mathrm{~L} 9+\mathrm{Q} 1+\mathrm{N} 3, \\
\mathrm{~L} 9+\mathrm{Q} 8+\mathrm{N} 1, & \mathrm{~L} 9+\mathrm{Q} 8+\mathrm{N} 3, \\
\mathrm{~L} 9+\mathrm{Q} 21+\mathrm{N} 1, & \mathrm{~L} 9+\mathrm{Q} 21+\mathrm{N} 3 .
\end{array}
$$

Some new complications arise in the analysis of these models. First, along with the KM matrix operating between the quark fields, we now have a lepton mixing matrix, $U_{e \nu}$, operating between the charged leptons and the neutrinos. Second, experiments to date have credited the neutrinos with either zero, or very small, masses - typically orders of magnitude less than for the other particles in a given generation. Table $\mathrm{V}$ shows the current bounds on the neutrino masses resulting from accelerator and double-beta decay measurements. This 
implies a disquietingly large disparity between the values of the Yukawa coupling constants in the mass terms for the neutrinos and the corresponding terms for the other fermion sectors. One way of avoiding this disparity is to invoke the see-saw mechanism [22 24] which allows for comparable Dirac masses for all the particles, but provides small observable Majorana masses for the neutrinos. Table $\square$ displays the results for the above models (with the exception of the model $L 9+Q 21+N 1$ which is the same as the SM with RHNs and no horizontal symmetry).

Since the charged fermion sectors have been dealt with, in studying these models all of the comments made in Sec. III will apply here also. Many of the cases can be disregarded because they suffer from problems in the quark sectors or the charged lepton sector. Others suffer from unfavourable KM matrix results. Those models that show promise after this initial filtering are then subject to analysis to see what they predict for the neutrino sector.

For several of these models, it will be seen that the RHNs do not couple to some of the possible higgs multiplets. In these cases the neutrinos remain massless at tree level, acquiring masses only - if at all — through radiative corrections, providing a natural reason for the small or zero masses observed for these particles.

For other cases, investigation is hampered by the lack of data concerning the precise masses of the neutrinos, and the values of the mixing angles between the two lepton sectors. Leaving aside the possibility that the neutrinos are, in fact, massless, the smallness of their masses can be explained through the so-called see-saw mechanism. Here, the neutrinos initially acquire Dirac masses of comparable size to the masses of the other fermion sectors. The RHN fields, however, also develop very large Majorana masses. The observed neutrino masses are then those resulting from diagonalising the matrix below:

$$
\mathcal{L}_{\text {mass }}=\left(\overline{\nu_{L}}, \overline{\left(\nu_{R}\right)^{C}}\right)\left(\begin{array}{cc}
0 & m \\
m^{T} & M
\end{array}\right)\left(\begin{array}{c}
\left(\nu_{L}\right)^{C} \\
\nu_{R}
\end{array}\right),
$$

where $m$ is a three-by-three matrix of Dirac masses, and $M$ is the corresponding matrix of Majorana masses. The eigenvalues for this matrix are, for $M$ large, $M$ (assumed to be unobserved as yet) and $m^{2} / M$. The latter value is the observed result, and can clearly be 
very small for large values of $M$. In general, for a similar spread of values, the Dirac masses should have more impact on the (observable) neutrino mass hierarchy as the latter depend on the difference of the squares of the Dirac masses, while only depending on the (linear) difference of the Majorana masses.

It is worth noting that the Majorana terms in the Lagrangian may arise from Yukawa couplings of the form

$$
\mathcal{L}_{\text {Yuk }}=\lambda \overline{\left(\nu_{R}\right)^{C}} \sigma \nu_{R}
$$

where $\sigma$ is a scalar under the standard model gauge group, but not necessarily under the horizontal gauge group. For example, if the RHNs transform as a triplet under $S U(2)_{H}$, then $\sigma$ could be either a $\underline{1}, \underline{3}$ or $\underline{5}$ under the horizontal gauge group. Such scalar fields are ideal both for breaking the horizontal symmetry at a high energy and for generating radiative corrections, as described in Sec. $\nabla$. Furthermore, the very high VEVs that these fields need to develop to fulfill this function automatically assure that the Majorana masses will be extremely high.

In the table, the neutrinos have been labelled $\nu_{1}, \nu_{2}$ and $\nu_{3}$, since it cannot be said what mixture of these mass eigenstates corresponds to the weak eigenstates, $\nu_{e}, \nu_{\mu}$ and $\nu_{\tau}$. This is, of course, because the nature of the lepton mixing matrix is largely unknown. If the offdiagonal entries of this matrix were large, however, then non-conservation of the individual lepton numbers $L_{e}, L_{\mu}$ and $L_{\tau}$ should be easy to observe. The lack of such observations particularly for $\nu_{e} \leftrightarrow \nu_{\mu}$ exchanges — places severe limits on these values. This suggests that, at least, $\nu_{1}$ is the principle component of the electron neutrino.

In order to further investigate any of the cases above, it becomes necessary to choose a particular model for the neutrino mass spectrum. Here, we shall look at a paper by Caldwell and Langacker [25]. They take as their starting point the $17 \mathrm{keV}$ neutrino claimed to exist by Simpson and others 26 32. By looking at the effects of various constraints, Caldwell and Langacker reached the following conclusions. Firstly, the $17 \mathrm{keV}$ neutrino is assumed to be $\nu_{3}$, and is found to be the dominant component of $\nu_{\tau}$. Secondly, $\nu_{\mu}$ is a heavy Majorana 
neutrino, with a mass of either about $17 \mathrm{keV}$ or in the range $170-270 \mathrm{keV}$.

For the case that the masses of $\nu_{2}$ and $\nu_{3}$ are both $17 \mathrm{keV}$, with the muon and tauon neutrinos being almost pure mixtures of these two mass eigenstates, a further symmetry is possible. Here, the mixing angles between the first and second and first and third generation leptons are small and equal, while the second-third generation angle, $\theta_{3}$, satisfies

$$
\cos \theta_{3}=\frac{1}{\sqrt{2}}=-\sin \theta_{3}
$$

This symmetry conserves $L_{e}-L_{\mu}+L_{\tau}$. Small deviations from this symmetry are possible, but the exact symmetry would be expected to show through at the tree level. One candidate model for such a symmetry is the $L_{3}+Q_{4}+N_{3}$ model with the $\zeta \sim \underline{4}$ higgs multiplet, which suffers from a second-third generation mixing angle hierarchy problem in the KM matrix, and does not explicitly equate the neutrino masses, but is otherwise suitable (perhaps a further relation, maybe coming from a more thorough analysis of the higgs potential, can restrict the model further, forcing the neutrino masses to be equal and giving the mixing angle its appropriate value). None of the other cases looks promising for this first scenario.

The second possibility found by Caldwell and Langacker has all the lepton mixing angles small, with $17 \mathrm{keV}$ for the mass of $\nu_{3}$, and hence for $\nu_{\tau}$, and 170-270 keV for the mass of $\nu_{2}$, which is the dominant component of $\nu_{\mu}$. It may be, in this case, that the muon neutrino mass is generated at tree level (thus engendering a name change in table $\mathrm{V}: \nu_{3} \leftrightarrow \nu_{2}$ ), while the $17 \mathrm{keV}$ mass arises from radiative corrections. Feasible models for generating this scenario are the $L_{7}+Q_{18}+N_{1}$ model with the $\phi$ higgs, transforming as a scalar under the horizontal symmetry; the $L_{4}+Q_{8}+N_{2}$ model with $\Delta \sim \underline{3}$ higgs multiplet (the $\chi$ Higgs multiplet in this model is investigated in Ref. [14], it provides tree level masses for the second and third generation, including neutrinos, but generates non-zero lepton mixing angles at tree level, which therefore may be quite large); the model $L_{3}+Q_{4}+N_{1}$, with $\phi$ higgs (here, all the fermion masses and mixing angles would need to be generated from mixing with $\nu_{2}$ through radiative corrections. This seems a little dubious, especially since one would expect the second generation particles to receive greater masses than the third generation. One 
way around this is to combine the $\phi$ Higgs with the $\Delta$ Higgs, which provides masses for the charged third generation particles t, b and $\tau)$; the $L_{3}+Q_{4}+N_{3}$ model with the $\Delta \sim \underline{3}$ higgs multiplet; and the $L_{9}+Q_{8}+N_{3}$ model with the $\Delta$ higgs multiplet (although the lack of a mass for the tauon at tree level is a little odd).

\section{OTHER ASPECTS OF $S U(2)_{H}$ MODELS}

Having examined the tree level results for models based on a horizontal $S U(2)$ gauge symmetry, some general points about such models are in order. Firstly, these models will generate flavour changing neutral currents (FCNCs) mediated by $S U(2)_{H}$ gauge bosons. FCNCs will also be generated in the Higgs sector. The FCNCs resulting from Higgs couplings can be suppressed by giving the Higgs bosons sufficiently large masses. Since the Higgs sectors we have studied serve only as starting points for more realistic mass generation schemes, we will not pursue any detailed Higgs phenomenology in the present paper. Note, however, the generic feature that the Higgs-induced FCNC processes will be weaker for the lighter fermions compared with the heavier fermions.

The gauge-boson generated FCNCs may be suppressed by breaking the horizontal symmetry at a high energy scale, thus generating large masses for the bosons. The following is a simplified, order-of-magnitude examination of minimum gauge boson masses due to constraints from the observed smallness of certain FCNC processes. More precise calculations of the mass limits would be cumbersome and of limited worth since either the minimum values for the gauge boson masses are found to be beyond the energies of any present-day operating or planned facility or other factors preclude the likely observation of the bosons (an example of such a case is included below). The horizontal gauge boson masses are assumed to be degenerate and mixing with the $\mathrm{Z}$ boson is assumed to be very small. This simplifies the discussion without seriously affecting the results. The actual mechanism for generating the horizontal gauge boson masses is outlined later in this section.

It is found that many models will not support various of the FCNC processes that might 
be considered. In particular, it is worth noting that the tree-level horizontal contribution to the $K_{S}-K_{L}$ mass difference will cancel out in every model, even when a tree-level Cabibbo angle is generated. The coupling here is of the form

$$
\lambda \bar{f}_{L, R} U^{\dagger} T^{a} U \gamma^{\mu} f_{L, R} H_{\mu}^{a}
$$

where $f_{L, R}$ is the mass eigenstate fermion field, $U$ is the unitary matrix rotating the weak eigenstates of the fermions into mass eigenstates, $T^{a}$ are the generators of the horizontal symmetry and $H^{a}$ are the associated horizontal bosons. If the same field $f_{L, R}$ couples to each end of the horizontal boson propagator, then it turns out that the angles (which exist because the Higgs multiplets and the fermion fields transform non-trivially under $\left.S U(2)_{H}\right)$ cancel out in the sum over $a$, resulting in a zero contribution to the process.

Hence, different processes will provide the best constraints on the horizontal boson masses for different models. In this paper, three different processes shall be looked at, chosen to exemplify the main types of processes to which the horizontal models can contribute.

First, the lepton-number violating decay

$$
\tau^{-} \rightarrow \mu^{-} e^{+} \mu^{-}
$$

shall be looked at. This decay occurs, for example, in the $\underline{L_{1}+Q_{8}}$ model with the Higgs multiplet and the leptonic fermion fields transforming as triplets under $S U(2)_{H}$. Fig. (11) shows a diagram of the decay for this model. For simplicity, the Lorentz form of the interactions will be ignored, with the result coming from a comparison with the branching ratio of a similar decay. For example, in this instance, the branching ratio of the lepton-number violating decay will be compared to that of the decay

$$
\tau^{-} \rightarrow \mu^{-} \nu_{\tau} \bar{\nu}_{\mu}
$$

Thus, one arrives at the expression $\left(g_{H}, M_{H}, g_{W}\right.$ and $M_{W}$ represent respectively the horizontal coupling constant and gauge boson mass, the weak coupling constant and the W boson mass) 


$$
\frac{g_{H}^{2}}{M_{H}^{2}} \leq \frac{\operatorname{BR}(\tau \rightarrow \mu e \mu) \times g_{W}^{2}}{\operatorname{BR}(\tau \rightarrow \mu \nu \bar{\nu}) \times M_{W}^{2}} .
$$

The experimental value for the $\mathrm{SM}$ decay is $\operatorname{BR}(\tau \rightarrow \mu \nu \bar{\nu})=0.178 \pm 0.004$ [33] while the limit on the lepton-number violating decay is $\operatorname{BR}\left(\tau \rightarrow \mu^{-} e^{+} \mu^{-}\right)<3.8 \times 10^{-5}$ [34, leading to the limit

$$
\frac{g_{H}^{2}}{M_{H}^{2}} \leq 2.18 \times 10^{-4} \times \frac{g_{W}^{2}}{M_{W}^{2}}
$$

In the case of the coupling constants being equal, the lower limit on the horizontal gauge boson mass becomes (taking $M_{W}=80.6 \mathrm{GeV}$ [35])

$$
M_{H} \gtrsim 5 \mathrm{TeV} \text {. }
$$

One of the most stringent limits on the gauge boson masses comes from the semi-leptonic decay

$$
K^{-} \rightarrow \pi^{-} e^{+} \mu^{-}
$$

which occurs, for example, in three of the six more promising models discussed in Sec. III. In these models the first two generations typically form doublet fields under the horizontal symmetry. In the $\underline{L_{7}+Q_{18}}$ model with $\phi \sim \underline{1}$ Higgs multiplet, the decay will proceed as shown in Fig. (2). In this case, we arrive at the approximate ratio

$$
\frac{g_{H}^{2}}{M_{H}^{2}} \leq \frac{g_{W}^{2} \times \sin ^{2} \theta_{c} \times \operatorname{BR}\left(K^{-} \rightarrow \pi^{-} e^{+} \mu^{-}\right)}{2 \times \operatorname{BR}\left(K^{-} \rightarrow \pi^{0} \mu^{-} \bar{\nu}_{\mu}\right) \times M_{W}^{2}}
$$

where $\theta_{c}$ is the Cabibbo angle.

Observations to-date [36] put the rate of the FCNC decay at

$$
\mathrm{BR}\left(K^{-} \rightarrow \pi^{-} e^{+} \mu^{-}\right)<2.1 \times 10^{-10}
$$

while the SM decay rate is 37

$$
\mathrm{BR}\left(K^{-} \rightarrow \pi^{0} \mu^{-} \bar{\nu}_{\mu}\right)=0.0318 \pm .0008
$$

leading to the bound 


$$
\frac{g_{H}^{2}}{M_{H}^{2}} \leq 1.69 \times 10^{-10} \times \frac{g_{W}^{2}}{M_{W}^{2}}
$$

or, for equal-valued coupling constants, we get the extreme mass limit

$$
M_{H} \gtrsim 6 \times 10^{3} \mathrm{TeV} .
$$

Finally, we take a look at the model $\underline{L_{9}+Q_{8}}$ with $\Delta \sim \underline{3}$ Higgs. This model proves interesting in that only the second and third generation quark fields transform non-trivially under the horizontal symmetry. Thus, the only allowed FCNC processes must involve mixing between either the top and charm quarks or the bottom and strange quarks. Thus the most extreme limits will come from processes such as the decay of the $\Upsilon$ meson into a $\phi$ meson (Fig. (3) shows one possible channel for this decay, with gluon emission from one of the final-state quarks for kinematic balance). The limit from decays such as this, though, will be very weak as little experimental information is available on the particles involved and the experimental uncertainties are comparatively large. While it is possible, then, that the horizontal bosons could have fairly low masses in such models, production and investigation of the bosons will be hampered by the lack of tree-level interactions between the horizontal bosons and the first-generation fermion fields.

Another concern of horizontal models of this kind is how to generate the radiative corrections that are assumed to provide the smaller masses and mixing angles in the models. What follows is a brief description of one method for achieving this 14,38.

First, a scalar field $(\sigma)$ is introduced that transforms as a singlet under the SM gauge group, but not under $S U(2)_{H}$. This is a neutral field and is assumed to pick up a large VEV, breaking the horizontal symmetry at a high energy scale. As well as leading to radiative corrections, this will generate the large masses for the horizontal gauge bosons discussed above. As has been noted in the previous section, such neutral scalars may appear anyway in the Majorana neutrino terms in the lagrangian. There, the large VEVs for these scalar fields leads to suitably large Majorana masses and the see-saw mechanism then naturally explains the smallness of observed neutrino masses. 
Further scalar fields are introduced $\left(\eta, \eta^{\prime}\right)$ which are charged (thus they acquire no VEVs and will not affect the tree level results) and which couple with the neutral scalar field, inducing mass mixing between them. These latter fields will also couple to the fermion fields, producing 1-loop diagrams such as that shown in Fig. (4) that will provide small corrections to the masses and mixing angles.

An interesting feature of many models with masses generated through radiative corrections is that they couple the up quark to the strange and bottom quarks and couple the down quark to the charm and top quarks through the higher-order corrections, thus providing a natural explanation for the observation $m_{u}<m_{d}$.

\section{CONCLUSION}

An $\mathrm{SU}(2)$ gauge symmetry between the three generations of fermions is one possibility for explaining the major features of the observed fermion mass and mixing angle spectrum.

If there are no RHN fields, then there are ten ways to assign representations under this symmetry to the different fermion fields such that gauge and global anomalies cancel. We have surveyed each of these models to determine which possible Higgs multiplets lead to promising tree level mass relations and KM matrices. A brief summary of the results for each model is given in table $\mathrm{IV}$.

In the case where RHN fields are also assumed to exist, a further 35 models satisfying anomaly cancellation are possible. While many of these models can be discarded as unlikely to represent nature because of problems in the charged fermion sectors, analysis of the predictions for neutrino masses is hindered by the lack of definite information regarding these masses. We investigated the models containing RHNs, taking as assumptions the existence of the claimed $17 \mathrm{keV}$ neutrino and following the study of Caldwell and Langacker into the consequences of this assumption.

For the future, there are various areas of work to follow up on. Having found the tree

level results for a given model, the next step is to work out the details of generating radiative 
corrections, for which a possible method is outlined in Sec. $\nabla$. Also, further phenomenology associated with horizontal $S U(2)$ symmetry models, such as the Higgs potentials - which may show how to improve on some of the models by preventing some of the neutral Higgs components from gaining VEVs - may be investigated.

We wish to thank Andrew Davies for some useful discussions. 


\section{REFERENCES}

[1] G. Ecker, Z. Phys. C 24 (1984) 353.

[2] R. Barbieri, R. Gatto and F. Strocchi, Phys. Lett. 74 B (1978) 344.

[3] K.S. Babu and Xiao-Gang He, Phys. Rev. D 36 (1987) 3484.

[4] A. Davidson, M. Koca and K.C. Wali, Phys. Rev. Lett. 43 (1979) 92.

[5] A. Davidson, M. Koca and K.C. Wali, Phys. Rev. D 20 (1979) 1195.

[6] A. Davidson and K.C. Wali, Phys. Rev. D 21 (1980) 787.

[7] T. Maehara and T. Yanagida, Prog. Theor. Phys. 60 (1978) 822.

[8] J. Chakrabarti, Phys. Rev. D 20 (1979) 2411.

[9] C.L. Ong, Phys. Rev. D 19 (1979) 2738.

[10] C.L. Ong, Phys. Rev. D 22 (1980) 2886.

[11] F. Wilczek and A. Zee, Phys. Rev. Lett. 42 (1979) 421.

[12] K. Bandyopadhyay and D. Choudhury, Phys. Rev. D 43 (1991) 1646.

[13] M. T. Yamawaki and W. W. Wada, Phys. Rev. D 43 (1991) 2432.

[14] R. Foot, G.C. Joshi, H. Lew and R.R. Volkas, Phys. Lett. B 226 (1989) 318.

[15] K. S. Babu and R.N. Mohapatra, Phys. Rev. D 43 (1991) 2278.

[16] T. Yanagida, Phys. Rev. D 20 (1979) 2986.

[17] E. Papantonopoulos and G. Zoupanos, Phys. Lett. 110 B (1982) 465.

[18] G. Zoupanos, Phys. Lett. 115 B (1982) 221.

[19] E. Papantonopoulos and G. Zoupanos, Z. Phys. C 16 (1983) 361.

[20] Particle Data Group, Phys. Lett. B 239 (1990) III61. 
[21] The LEP Collaborations: ALEPH, DELPHI, L3, and OPAL, Phys. Lett. B 276 (1992) 247.

[22] M. Gell-Mann, P. Raymond and R. Slansky, in: Supergravity, eds. P. van Nieuwenhuizen and D.Z. Freedman (North-Holland, Amsterdam, 1979) p.315.

[23] T. Yanagida, in Proceedings of the Workshop on Unified Theory and Baryon Number in the Universe edited by A. Sawada and H. Sugawara (KEK, Tsukuba-Gun, Ibaraki-ken, Japan, 1979).

[24] R. N. Mohapatra and G. Senjanovic, Phys. Rev. Lett. 44 (1980) 912.

[25] D. O. Caldwell and P. Langacker, Phys. Rev. D 44 (1991) 823.

[26] J. J. Simpson, Phys. Rev. Lett. 54 (1985) 1891.

[27] J. J. Simpson and A. Hime, Phys. Rev. D 39 (1989) 1825.

[28] A. Hime and J. J. Simpson, Phys. Rev. D 39 (1989) 1837.

[29] B. Sur et al., Phys. Rev. Lett. 66 (1991) 2444.

[30] E. B. Norman et al., J. Phys. G 17 (1991) Suppl. S291.

[31] I. Zliman et al., Phys. Rev. Lett. 67 (1991) 560.

[32] A. Hime and N. A. Jelley, Phys. Lett. B 257 (1991) 441.

[33] Particle Data Group, Phys. Lett. B 239 (1990) VI15.

[34] Particle Data Group, Albrecht et al., Phys. Lett. B 239 (1990) VI16.

[35] Particle Data Group, Phys. Lett. B 239 (1990) V1.

[36] Particle Data Group, Lee et al., Phys. Lett. B 239 (1990) VII71.

[37] Particle Data Group, Chiang et al., Phys. Lett. B 239 (1990) VII71.

[38] B.S. Balakrishna, Phys. Rev. Lett. 60 (1988) 1602. 
[39] Particle Data Group, Cohen et al., Blocker et al., Phys. Lett. B 239 (1990) II2.

[40] Particle Data Group, Dominguez et al., Gasser et al. and Narison, Phys. Lett. B 239 (1990) III56. 


\section{FIGURES}

FIG. 1. Horizontal gauge boson induced, lepton number violating decay.

FIG. 2. Horizontal gauge boson induced kaon decay.

FIG. 3. Horizontal gauge boson induced Upsilon decay. While gluon emission by one of the final-state quarks will dominate, other emissions such as photon or Z boson emission are also possible.

FIG. 4. A one-loop level diagram of the type that leads to masses for the lighter fermions. Here, an initially massless fermion $\left(\mathrm{f}_{L}, \mathrm{f}_{R}\right)$ is converted to and from a heavy fermion $\left(\mathrm{F}_{L}, \mathrm{~F}_{R}\right)$ through the emission of charged scalars $\left(\eta, \eta^{\prime}\right)$. These scalars couple via a neutral scalar coming out of the vacuum $(\langle\sigma\rangle)$. Thus the light fermion will pick up a mass which is dependent on the mass of the

heavy fermion $\left(m_{F}\right)$, the VEV of the neutral scalar, the masses of the charged scalars and the Yukawa coupling constants of the charged scalars. 


\section{TABLES}

TABLE I. Observed Masses of Fundamental Particles [39,40]

\begin{tabular}{crrrrr}
\hline \hline$m_{\text {up }}$ & $\sim 5.6 \pm 1.1 \mathrm{MeV}$ & $m_{\text {charm }}$ & $\sim 1.35 \pm 0.05 \mathrm{GeV}$ & $m_{\text {top }}$ & $>89 \mathrm{GeV}$ \\
$m_{\text {down }}$ & $\sim 9.9 \pm 1.1 \mathrm{MeV}$ & $m_{\text {strange }}$ & $\sim 199 \pm 33 \mathrm{MeV}$ & $m_{\text {bottom }}$ & $\sim 5 \mathrm{GeV}$ \\
$m_{\text {elec }}$ & $0.511 \mathrm{MeV}$ & $m_{\text {muon }}$ & $105.7 \mathrm{MeV}$ & $m_{\text {tauon }}$ & $1784.1_{-3.6}^{+2.7} \mathrm{MeV}$ \\
\hline \hline
\end{tabular}

TABLE II. Possible Lepton $S U(2)_{H}$ Representation Assignments

\begin{tabular}{lcccc}
\hline \hline & $l_{L}$ & $e_{R}$ & Gauge Anomaly & Global Anomaly \\
(\# of doublets)
\end{tabular}


TABLE III. Possible Quark $S U(2)_{H}$ Representation Assignments

\begin{tabular}{|c|c|c|c|c|c|}
\hline & $q_{L}$ & $u_{R}$ & $d_{R}$ & $\begin{array}{c}\text { Gauge Anomaly } \\
\text { Contribution }\end{array}$ & $\begin{array}{l}\text { Global Anomaly } \\
\text { (\# of doublets) }\end{array}$ \\
\hline$Q_{1}$ & $\underline{3}$ & $\underline{3}$ & $\underline{3}$ & 0 & 0 \\
\hline$Q_{2}$ & $\underline{3}$ & $\underline{2} \oplus \underline{1}$ & $\underline{3}$ & 12 & 3 \\
\hline$Q_{3}$ & $\underline{3}$ & $\underline{3}$ & $\underline{2} \oplus \underline{1}$ & -6 & 3 \\
\hline$Q_{4}$ & $\underline{2} \oplus \underline{1}$ & $\underline{3}$ & $\underline{3}$ & -6 & 6 \\
\hline$Q_{5}$ & $\underline{3}$ & $\underline{2} \oplus \underline{1}$ & $\underline{2} \oplus \underline{1}$ & 6 & 6 \\
\hline$Q_{6}$ & $\underline{2} \oplus \underline{1}$ & $\underline{2} \oplus \underline{1}$ & $\underline{3}$ & 6 & 9 \\
\hline$Q_{7}$ & $\underline{2} \oplus \underline{1}$ & $\underline{3}$ & $\underline{2} \oplus \underline{1}$ & -12 & 9 \\
\hline$Q_{8}$ & $\underline{2} \oplus \underline{1}$ & $\underline{2} \oplus \underline{1}$ & $\underline{2} \oplus \underline{1}$ & 0 & 12 \\
\hline$Q_{9}$ & $\underline{3}$ & $\underline{1} \oplus \underline{1} \oplus \underline{1}$ & $\underline{3}$ & 16 & 0 \\
\hline$Q_{10}$ & $\underline{3}$ & $\underline{3}$ & $\underline{1} \oplus \underline{1} \oplus \underline{1}$ & -8 & 0 \\
\hline$Q_{11}$ & $\underline{1} \oplus \underline{1} \oplus \underline{1}$ & $\underline{3}$ & $\underline{3}$ & -8 & 0 \\
\hline$Q_{12}$ & $\underline{3}$ & $\underline{1} \oplus \underline{1} \oplus \underline{1}$ & $\underline{1} \oplus \underline{1} \oplus \underline{1}$ & 8 & 0 \\
\hline$Q_{13}$ & $\underline{1} \oplus \underline{1} \oplus \underline{1}$ & $\underline{1} \oplus \underline{1} \oplus \underline{1}$ & $\underline{3}$ & 8 & 0 \\
\hline$Q_{14}$ & $\underline{1} \oplus \underline{1} \oplus \underline{1}$ & $\underline{3}$ & $\underline{1} \oplus \underline{1} \oplus \underline{1}$ & -16 & 0 \\
\hline
\end{tabular}




\begin{tabular}{|c|c|c|c|c|c|}
\hline$Q_{15}$ & $\underline{2} \oplus \underline{1}$ & $\underline{1} \oplus \underline{1} \oplus \underline{1}$ & $\underline{2} \oplus \underline{1}$ & 4 & 9 \\
\hline$Q_{16}$ & $\underline{2} \oplus \underline{1}$ & $\underline{2} \oplus \underline{1}$ & $\underline{1} \oplus \underline{1} \oplus \underline{1}$ & -2 & 9 \\
\hline$Q_{17}$ & $\underline{1} \oplus \underline{1} \oplus \underline{1}$ & $\underline{2} \oplus \underline{1}$ & $\underline{2} \oplus \underline{1}$ & -2 & 6 \\
\hline$Q_{18}$ & $\underline{2} \oplus \underline{1}$ & $\underline{1} \oplus \underline{1} \oplus \underline{1}$ & $\underline{1} \oplus \underline{1} \oplus \underline{1}$ & 2 & 6 \\
\hline$Q_{19}$ & $\underline{1} \oplus \underline{1} \oplus \underline{1}$ & $\underline{1} \oplus \underline{1} \oplus \underline{1}$ & $\underline{2} \oplus \underline{1}$ & 2 & 3 \\
\hline$Q_{20}$ & $\underline{1} \oplus \underline{1} \oplus \underline{1}$ & $\underline{2} \oplus \underline{1}$ & $\underline{1} \oplus \underline{1} \oplus \underline{1}$ & -4 & 3 \\
\hline$Q_{21}$ & $\underline{1} \oplus \underline{1} \oplus \underline{1}$ & $\underline{1} \oplus \underline{1} \oplus \underline{1}$ & $\underline{1} \oplus \underline{1} \oplus \underline{1}$ & 0 & 0 \\
\hline$Q_{22}$ & $\underline{3}$ & $\underline{2} \oplus \underline{1}$ & $\underline{1} \oplus \underline{1} \oplus \underline{1}$ & 4 & 3 \\
\hline$Q_{23}$ & $\underline{3}$ & $\underline{1} \oplus \underline{1} \oplus \underline{1}$ & $\underline{2} \oplus \underline{1}$ & 10 & 3 \\
\hline$Q_{24}$ & $\underline{2} \oplus \underline{1}$ & $\underline{3}$ & $\underline{1} \oplus \underline{1} \oplus \underline{1}$ & -14 & 6 \\
\hline$Q_{25}$ & $\underline{2} \oplus \underline{1}$ & $\underline{1} \oplus \underline{1} \oplus \underline{1}$ & $\underline{3}$ & 10 & 6 \\
\hline$Q_{26}$ & $\underline{1} \oplus \underline{1} \oplus \underline{1}$ & $\underline{3}$ & $\underline{2} \oplus \underline{1}$ & -14 & 3 \\
\hline$Q_{27}$ & $\underline{1} \oplus \underline{1} \oplus \underline{1}$ & $\underline{2} \oplus \underline{1}$ & $\underline{3}$ & 4 & 3 \\
\hline
\end{tabular}


TABLE IV. Summary of Tree Level Results.

\begin{tabular}{|c|c|c|c|}
\hline Models & Higgs & Mass Relations & KM Matrix \\
\hline \multirow[t]{3}{*}{$L_{8}+Q_{16}$} & $\phi \sim 1$ & masses for $\mathrm{u}, \mathrm{c}, \mathrm{t}$ (two being equal); $\mathrm{b} ; \tau$ & $U_{K M}=I_{3 \times 3}$ \\
\hline & $\chi \sim \underline{2}$ & masses for $\mathrm{c}, \mathrm{t} ; \mathrm{b} ; \tau$ & 1-3 mixing angle \\
\hline & $\Delta \sim \underline{3}$ & masses for $\mathrm{c}, \mathrm{t}$ & unphysical \\
\hline \multirow[t]{2}{*}{$L_{7}+Q_{18}$} & $\phi \sim 1$ & masses for $\mathrm{t} ; \mathrm{b} ; \tau$ & $U_{K M}=I_{3 \times 3}$ \\
\hline & $\chi \sim \underline{2}$ & masses for $\mathrm{t} ; \mathrm{b} ; \tau$ & 2-3 mixing angle \\
\hline$L_{6}+Q_{11}$ & $\Delta \sim \underline{3}$ & masses for $\mathrm{t} ; \mathrm{b} ; \tau$ & $1-3$ and $2-3$ mixing angles \\
\hline \multirow[t]{3}{*}{$L_{6}+Q_{10}$} & $\phi \sim 1$ & $m_{u}=m_{c}=m_{t}$ & unphysical \\
\hline & $\Delta \sim \underline{3}$ & masses for $\mathrm{c}, \mathrm{t} ; \mathrm{b} ; \tau$ & zero diagonal entries \\
\hline & $\psi \sim \underline{5}$ & masses for $\mathrm{u}, \mathrm{c}, \mathrm{t}$ & unphysical \\
\hline \multirow[t]{2}{*}{$L_{5}+Q_{13}$} & $\phi \sim 1$ & masses for $\mathrm{u}, \mathrm{c}, \mathrm{t}$ & unphysical \\
\hline & $\Delta \sim \underline{3}$ & masses for $\mathrm{b} ; \tau$ & unphysical \\
\hline$L_{5}+Q_{12}$ & $\Delta \sim \underline{3}$ & masses for $\mathrm{t} ; \mathrm{b} ; \tau$ & $1-3$ and $2-3$ mixing angles \\
\hline \multirow[t]{3}{*}{$L_{3}+Q_{4}$} & $\chi \sim \underline{2}$ & $m_{c}=\frac{m_{t}}{\sqrt{2}}, m_{s}=\frac{m_{b}}{\sqrt{2}}, m_{\mu}=\frac{m_{\tau}}{\sqrt{2}}$ & 2-3 mixing angle \\
\hline & $\Delta \sim \underline{3}$ & masses for $\mathrm{t} ; \mathrm{b} ; \tau$ & $U_{K M}=I_{3 \times 3}$ \\
\hline & $\zeta \sim \underline{4}$ & masses for $\mathrm{c}, \mathrm{t} ; \mathrm{s}, \mathrm{b} ; \mu, \tau$ & 2-3 mixing angle \\
\hline \multirow[t]{4}{*}{$L_{2}+Q_{6}$} & $\phi \sim \underline{1}$ & masses for $\mathrm{u}, \mathrm{c}, \mathrm{t}$ - two masses equal & unphysical \\
\hline & $\chi \sim \underline{2}$ & $m_{s}=\frac{m_{b}}{\sqrt{2}}, m_{\mu}=\frac{m_{\tau}}{\sqrt{2}}$, masses for $\mathrm{c}, \mathrm{t}$ & zero diagonal entries \\
\hline & $\Delta \sim \underline{3}$ & masses for $\mathrm{c}, \mathrm{t} ; \mathrm{b} ; \tau$ & 2-3 mixing angle \\
\hline & $\zeta \sim \underline{4}$ & masses for $\mathrm{s}, \mathrm{b} ; \mu, \tau$ & unphysical \\
\hline
\end{tabular}




\begin{tabular}{|c|c|c|c|}
\hline \multirow[t]{4}{*}{$L_{1}+Q_{8}$} & $\phi \sim \underline{1}$ & all massive, many equal masses & $U_{K M}=I_{3 \times 3}$ \\
\hline & $\chi \sim \underline{2}$ & masses for $\mathrm{c}, \mathrm{t} ; \mathrm{s}, \mathrm{b}$ & Cabibbo angle \\
\hline & $\Delta \sim \underline{3}$ & masses for $\mathrm{c}, \mathrm{t} ; \mathrm{s}, \mathrm{b} ; \tau$ & $U_{K M}=I_{3 \times 3}$ \\
\hline & $\psi \sim \underline{5}$ & masses for e, $\mu, \tau$ & unphysical \\
\hline \multirow[t]{3}{*}{$L_{1}+Q_{1}$} & $\phi \sim \underline{1}$ & three equal masses for each sector & $U_{K M}=I_{3 \times 3}$ \\
\hline & $\Delta \sim \underline{3}$ & masses for $\mathrm{t} ; \mathrm{b} ; \tau$ & $1-3$ and $2-3$ mixing angles \\
\hline & $\psi \sim \underline{5}$ & all massive & all entries non-zero \\
\hline \multirow[t]{3}{*}{$L_{9}+Q_{8}$} & $\phi \sim \underline{1}$ & all massive, some equal & $U_{K M}=I_{3 \times 3}$ \\
\hline & $\chi \sim \underline{2}$ & masses for $\mathrm{c}, \mathrm{t} ; \mathrm{s}, \mathrm{b}$ & Cabibbo angle \\
\hline & $\Delta \sim \underline{3}$ & masses for $\mathrm{c}, \mathrm{t} ; \mathrm{s}, \mathrm{b}$ & $U_{K M}=I_{3 \times 3}$ \\
\hline \multirow[t]{3}{*}{$L_{9}+Q_{1}$} & $\phi \sim \underline{1}$ & all massive, many equal masses & $U_{K M}=I_{3 \times 3}$ \\
\hline & $\Delta \sim \underline{3}$ & masses for $\mathrm{t} ; \mathrm{b}$ & $1-3$ and $2-3$ mixing angles \\
\hline & $\psi \sim \underline{5}$ & masses for all quarks & all entries non-zero \\
\hline \multirow[t]{3}{*}{$L_{1}+Q_{21}$} & $\phi \sim \underline{1}$ & all massive, $m_{e}=m_{\mu}=m_{\tau}$ & all entries non-zero \\
\hline & $\Delta \sim \underline{3}$ & mass for $\tau$ only & unphysical \\
\hline & $\psi \sim \underline{5}$ & masses for e, $\mu, \tau$ & unphysical \\
\hline
\end{tabular}


TABLE V. Summary of Results for Models with RHNs.

\begin{tabular}{|c|c|c|c|c|}
\hline Models & Higgs & Mass Relations & KM Matrix & Lepton Matrix \\
\hline \multirow[t]{4}{*}{$L_{8}+Q_{17}+N_{2}$} & \multirow[t]{2}{*}{$\phi \sim 1$} & \multirow[t]{2}{*}{ masses for $\mathrm{t} ; \mathrm{b} ; \tau ; \nu_{3}$} & $1-3$ and $2-3$ & $1-3$ and $2-3$ \\
\hline & & & mixing angles & mixing angles \\
\hline & \multirow[t]{2}{*}{$\chi \sim \underline{2}$} & \multirow[t]{2}{*}{ masses for $\mathrm{t} ; \mathrm{b} ; \tau ; \nu_{3}$} & $1-3$ and $2-3$ & $1-3$ and $2-3$ \\
\hline & & & mixing angles & mixing angles \\
\hline \multirow[t]{4}{*}{$L_{8}+Q_{16}+N_{1}$} & $\phi \sim \underline{1}$ & masses for $\mathrm{u}, \mathrm{c}, \mathrm{t}$ & $U_{K M}=I_{3 \times 3}$ & all entries \\
\hline & & (two being equal); b; $\tau$; all $\nu$ 's & & non-zero \\
\hline & $\chi \sim \underline{2}$ & masses for $\mathrm{c}, \mathrm{t} ; \mathrm{b} ; \tau$ & 1-3 mixing angle & unphysical \\
\hline & $\Delta \sim \underline{3}$ & masses for $\mathrm{c}, \mathrm{t}$ & unphysical & unphysical \\
\hline \multirow[t]{4}{*}{$L_{8}+Q_{16}+N_{3}$} & $\phi \sim 1$ & masses for $\mathrm{u}, \mathrm{c}, \mathrm{t}$ & $U_{K M}=I_{3 \times 3}$ & unphysical \\
\hline & & (two being equal); $\mathrm{b} ; \tau$ & & \\
\hline & $\chi \sim \underline{2}$ & masses for $\mathrm{c}, \mathrm{t} ; \mathrm{b} ; \tau$ & 1-3 mixing angle & unphysical \\
\hline & $\Delta \sim \underline{3}$ & masses for $\mathrm{c}, \mathrm{t} ; \nu_{3}$ & unphysical & unphysical \\
\hline \multirow[t]{4}{*}{$L_{7}+Q_{19}+N_{2}$} & $\phi \sim \underline{1}$ & masses for $\mathrm{u}, \mathrm{c}, \mathrm{t}$ & all entries & $U_{e \nu}=I_{3 \times 3}$ \\
\hline & & $\mathrm{b} ; \tau ; \nu_{1}=\nu_{2}=\nu_{3}$ & non-zero & \\
\hline & $\chi \sim \underline{2}$ & masses for $\mathrm{b} ; \tau ; \nu_{2}, \nu_{3}$ & unphysical & 1-3 mixing angle \\
\hline & $\Delta \sim \underline{3}$ & masses for $\nu_{2}, \nu_{3}$ & unphysical & unphysical \\
\hline \multirow{3}{*}{$L_{7}+Q_{18}+N_{1}$} & $\phi \sim \underline{1}$ & masses for $\mathrm{t} ; \mathrm{b} ; \tau ; \nu_{3}$ & $U_{K M}=I_{3 \times 3}$ & $U_{e \nu}=I_{3 \times 3}$ \\
\hline & $\chi \sim 2$ & masses for $\mathrm{t} ; \mathrm{b} ; \tau ; \nu_{3}$ & $2-3$ mixing angle & $1-3$ or $2-3$ \\
\hline & & & & mixing angle \\
\hline \multirow[t]{2}{*}{$L_{7}+Q_{18}+N_{3}$} & $\phi \sim \underline{1}$ & masses for $\mathrm{t} ; \mathrm{b} ; \tau$ & $U_{K M}=I_{3 \times 3}$ & unphysical \\
\hline & $\chi \sim \underline{2}$ & $\begin{array}{l}\text { masses for } \mathrm{t} ; \mathrm{b} ; \tau \\
\qquad m_{\nu_{2}}=\frac{m_{\nu_{3}}}{\sqrt{2}}\end{array}$ & 2-3 mixing angle & 2-3 mixing angle \\
\hline \multirow[t]{3}{*}{$L_{6}+Q_{11}+N_{1}$} & $\phi \sim \underline{1}$ & all $\nu$ 's massive & unphysical & unphysical \\
\hline & $\Delta \sim \underline{3}$ & masses for $\mathrm{t} ; \mathrm{b} ; \tau$ & $1-3$ and $2-3$ & unphysical \\
\hline & & & mixing angles & \\
\hline \multirow[t]{2}{*}{$L_{6}+Q_{11}+N_{3}$} & $\Delta \sim \underline{3}$ & masses for $\mathrm{t} ; \mathrm{b} ; \tau ; \nu_{3}$ & $1-3$ and $2-3$ & $1-3$ and $2-3$ \\
\hline & & & mixing angles & mixing angles \\
\hline \multirow[t]{5}{*}{$L_{6}+Q_{10}+N_{1}$} & $\phi \sim \underline{1}$ & $m_{u}=m_{c}=m_{t}$ & unphysical & unphysical \\
\hline & & all neutrinos massive & & \\
\hline & $\Delta \sim \underline{3}$ & masses for $\mathrm{c}, \mathrm{t} ; \mathrm{b} ; \tau$ & zero diagonal & unphysical \\
\hline & & & entries & \\
\hline & $\psi \sim \underline{5}$ & masses for $\mathrm{u}, \mathrm{c}, \mathrm{t}$ & unphysical & unphysical \\
\hline
\end{tabular}




\begin{tabular}{|c|c|c|c|c|}
\hline \multirow[t]{3}{*}{$L_{6}+Q_{10}+N_{3}$} & $\phi \sim \underline{1}$ & $m_{u}=m_{c}=m_{t}$ & unphysical & unphysical \\
\hline & $\Delta \sim \underline{3}$ & masses for $\mathrm{c}, \mathrm{t} ; \mathrm{b} ; \tau ; \nu_{3}$ & $\begin{array}{l}\text { zero diagonal } \\
\text { entries }\end{array}$ & $\begin{array}{l}1-3 \text { and } 2-3 \\
\text { mixing angles }\end{array}$ \\
\hline & $\psi \sim \underline{5}$ & masses for $\mathrm{u}, \mathrm{c}, \mathrm{t}$ & unphysical & unphysical \\
\hline \multirow[t]{2}{*}{$L_{5}+Q_{13}+N_{1}$} & $\phi \sim \underline{1}$ & masses for $\mathrm{u}, \mathrm{c}, \mathrm{t}$ & unphysical & unphysical \\
\hline & $\Delta \sim \underline{3}$ & masses for $\mathrm{b} ; \tau ; \nu_{3}$ & unphysical & $\begin{array}{l}1-3 \text { and } 2-3 \\
\text { mixing angles }\end{array}$ \\
\hline \multirow[t]{2}{*}{$L_{5}+Q_{13}+N_{3}$} & $\phi \sim \underline{1}$ & $\begin{array}{l}\text { masses for } \mathrm{u}, \mathrm{c}, \mathrm{t} \\
3 \text { equal } \nu \text { masses }\end{array}$ & unphysical & unphysical \\
\hline & $\Delta \sim \underline{3}$ & masses for $\mathrm{b} ; \tau ; \nu_{2}, \nu_{3}$ & unphysical & $\begin{array}{c}\text { zero diagonal } \\
\text { entries }\end{array}$ \\
\hline$L_{5}+Q_{12}+N_{1}$ & $\Delta \sim \underline{3}$ & masses for $\mathrm{t} ; \mathrm{b} ; \tau ; \nu_{3}$ & $\begin{array}{l}1-3 \text { and } 2-3 \\
\text { mixing angles }\end{array}$ & $\begin{array}{l}1-3 \text { and } 2-3 \\
\text { mixing angles }\end{array}$ \\
\hline \multirow{3}{*}{$L_{5}+Q_{12}+N_{3}$} & $\phi \sim \underline{1}$ & 3 equal $\nu$ masses & unphysical & unphysical \\
\hline & $\Delta \sim \underline{3}$ & masses for $\mathrm{t} ; \mathrm{b} ; \tau ; \nu_{2}, \nu_{3}$ & $\begin{array}{l}1-3 \text { and } 2-3 \\
\text { mixing angles }\end{array}$ & $\begin{array}{c}\text { zero diagonal } \\
\text { entries }\end{array}$ \\
\hline & $\psi \sim \underline{5}$ & $3 \nu$ masses & unphysical & unphysical \\
\hline \multirow[t]{3}{*}{$L_{4}+Q_{8}+N_{2}$} & $\phi \sim \underline{1}$ & $\begin{array}{c}\text { all massive, } \\
\text { many equal masses }\end{array}$ & $U_{K M}=I_{3 \times 3}$ & $U_{e \nu}=I_{3 \times 3}$ \\
\hline & $\chi \sim \underline{2}$ & masses for $\mathrm{c}, \mathrm{t} ; \mathrm{s}, \mathrm{b} ; \mu, \tau ; \nu_{2}, \nu_{3}$ & Cabibbo angle & $\begin{array}{c}1-2 \text { or } 1-3 \\
\text { mixing angle }\end{array}$ \\
\hline & $\Delta \sim \underline{3}$ & masses for $\mathrm{c}, \mathrm{t} ; \mathrm{s}, \mathrm{b} ; \mu, \tau ; \nu_{2}, \nu_{3}$ & $U_{K M}=I_{3 \times 3}$ & $U_{e \nu}=I_{3 \times 3}$ \\
\hline \multirow[t]{4}{*}{$L_{4}+Q_{1}+N_{2}$} & $\phi \sim \underline{1}$ & $\begin{array}{c}\text { all massive, } \\
\text { many equal masses }\end{array}$ & $U_{K M}=I_{3 \times 3}$ & $U_{e \nu}=I_{3 \times 3}$ \\
\hline & $\chi \sim \underline{2}$ & masses for $\mu, \tau ; \nu_{2}, \nu_{3}$ & unphysical & $\begin{array}{c}1-2 \text { or } 1-3 \\
\text { mixing angle }\end{array}$ \\
\hline & $\Delta \sim \underline{3}$ & masses for $\mathrm{t} ; \mathrm{b} ; \mu, \tau ; \nu_{2}, \nu_{3}$ & $\begin{array}{l}1-3 \text { and } 2-3 \\
\text { mixing angles }\end{array}$ & $\overline{U_{e \nu}=I_{3 \times 3}}$ \\
\hline & $\psi \sim \underline{5}$ & masses for $\mathrm{u}, \mathrm{c}, \mathrm{t} ; \mathrm{d}, \mathrm{s}, \mathrm{b}$ & $\begin{array}{l}\text { all entries } \\
\text { non-zero }\end{array}$ & unphysical \\
\hline \multirow[t]{4}{*}{$L_{3}+Q_{4}+N_{1}$} & $\phi \sim \underline{1}$ & mass for $\nu_{3}$ only & unphysical & unphysical \\
\hline & $\chi \sim \underline{2}$ & $\begin{array}{c}m_{c}=\frac{m_{t}}{\sqrt{2}} ; m_{s}=\frac{m_{b}}{\sqrt{2}} \\
m_{\mu}=\frac{m_{\tau}}{\sqrt{2}} ; \text { mass for } \nu_{3}\end{array}$ & $2-3$ mixing angle & $\begin{array}{c}1-3 \text { or } 2-3 \\
\text { mixing angle } \\
\end{array}$ \\
\hline & $\Delta \sim \underline{3}$ & masses for $\mathrm{t} ; \mathrm{b} ; \tau$ & $U_{K M}=I_{3 \times 3}$ & unphysical \\
\hline & $\zeta \sim \underline{4}$ & masses for $\mathrm{c}, \mathrm{t} ; \mathrm{s}, \mathrm{b} ; \mu, \tau$ & $2-3$ mixing angle & unphysical \\
\hline
\end{tabular}




\begin{tabular}{|c|c|c|c|c|}
\hline \multirow[t]{3}{*}{$L_{3}+Q_{4}+N_{3}$} & $\chi \sim \underline{2}$ & $\begin{array}{c}m_{c}=\frac{m_{t}}{\sqrt{2}} ; m_{s}=\frac{m_{b}}{\sqrt{2}} \\
m_{\mu}=\frac{m_{\tau}}{\sqrt{2}} ; m_{\nu_{2}}=\frac{m_{\nu_{3}}}{\sqrt{2}}\end{array}$ & $2-3$ mixing angle & $2-3$ mixing angle \\
\hline & $\Delta \sim \underline{3}$ & masses for $\mathrm{t} ; \mathrm{b} ; \tau ; \nu_{3}$ & $U_{K M}=I_{3 \times 3}$ & $U_{e \nu}=I_{3 \times 3}$ \\
\hline & $\zeta \sim \underline{4}$ & masses for $\mathrm{c}, \mathrm{t} ; \mathrm{s}, \mathrm{b} ; \mu, \tau ; \nu_{2}, \nu_{3}$ & $2-3$ mixing angle & 2-3 mixing angle \\
\hline \multirow[t]{5}{*}{$L_{3}+Q_{3}+N_{2}$} & $\phi \sim 1$ & $\begin{array}{c}m_{u}=m_{c}=m_{t} \\
3 \nu \text { masses - two equal }\end{array}$ & unphysical & unphysical \\
\hline & $\chi \sim \underline{2}$ & $\begin{array}{c}m_{s}=\frac{m_{b}}{\sqrt{2}} ; m_{\mu}=\frac{m_{\tau}}{\sqrt{2}} \\
\text { masses for } \nu_{2}, \nu_{3}\end{array}$ & unphysical & $\begin{array}{c}\text { zero diagonal } \\
\text { entries }\end{array}$ \\
\hline & $\Delta \sim \underline{3}$ & masses for $\mathrm{t} ; \mathrm{b} ; \tau ; \nu_{2}, \nu_{3}$ & $\begin{array}{l}1-3 \text { and } 2-3 \\
\text { mixing angles } \\
\end{array}$ & $\begin{array}{c}\text { all entries } \\
\text { non-zero }\end{array}$ \\
\hline & $\zeta \sim \underline{4}$ & masses for $\mathrm{s}, \mathrm{b} ; \mu, \tau$ & unphysical & unphysical \\
\hline & $\psi \sim \underline{5}$ & masses for $\mathrm{u}, \mathrm{c}, \mathrm{t}$ & unphysical & unphysical \\
\hline \multirow[t]{4}{*}{$L_{2}+Q_{6}+N_{1}$} & $\phi \sim 1$ & $\begin{array}{l}\text { masses for } \mathrm{u}, \mathrm{c}, \mathrm{t}- \\
\text { two masses equal }\end{array}$ & unphysical & unphysical \\
\hline & $\chi \sim \underline{2}$ & $\begin{array}{c}m_{s}=\frac{m_{b}}{\sqrt{2}}, m_{\mu}=\frac{m_{\tau}}{\sqrt{2}} \\
\text { masses for } \mathrm{c}, \mathrm{t}\end{array}$ & $\begin{array}{c}\text { zero diagonal } \\
\text { entries }\end{array}$ & unphysical \\
\hline & $\Delta \sim \underline{3}$ & masses for $\mathrm{c}, \mathrm{t} ; \mathrm{b} ; \tau ; \nu_{3}$ & $2-3$ mixing angle & $\begin{array}{c}1-3 \text { and } 2-3 \\
\text { mixing angles } \\
\end{array}$ \\
\hline & $\zeta \sim \underline{4}$ & masses for $\mathrm{s}, \mathrm{b} ; \mu, \tau$ & unphysical & unphysical \\
\hline \multirow[t]{5}{*}{$L_{2}+Q_{6}+N_{3}$} & $\phi \sim 1$ & $\begin{array}{c}\text { masses for } \mathrm{u}, \mathrm{c}, \mathrm{t} \text { (two equal); } \\
3 \text { equal } \nu \text { masses }\end{array}$ & unphysical & unphysical \\
\hline & $\chi \sim \underline{2}$ & $\begin{array}{c}m_{s}=\frac{m_{b}}{\sqrt{2}}, m_{\mu}=\frac{m_{\tau}}{\sqrt{2}} \\
\text { masses for } \mathrm{c}, \mathrm{t}\end{array}$ & $\begin{array}{c}\text { zero diagonal } \\
\text { entries } \\
\end{array}$ & unphysical \\
\hline & $\Delta \sim \underline{3}$ & masses for $\mathrm{c}, \mathrm{t} ; \mathrm{b} ; \tau ; \nu_{3}$ & $2-3$ mixing angle & $\begin{array}{l}1-3 \text { and } 2-3 \\
\text { mixing angles }\end{array}$ \\
\hline & $\zeta \sim \underline{4}$ & masses for $\mathrm{s}, \mathrm{b} ; \mu, \tau$ & unphysical & unphysical \\
\hline & $\psi \sim \underline{5}$ & $3 \nu$ masses & unphysical & unphysical \\
\hline \multirow[t]{3}{*}{$L_{2}+Q_{5}+N_{2}$} & $\chi \sim \underline{2}$ & $\begin{array}{c}m_{c}=\frac{m_{t}}{\sqrt{2}} ; m_{s}=\frac{m_{b}}{\sqrt{2}} \\
m_{\mu}=\frac{m_{\tau}}{\sqrt{2}} ; m_{\nu_{2}}=\frac{m_{\nu_{3}}}{\sqrt{2}}\end{array}$ & $\begin{array}{l}\text { zero diagonal } \\
\text { entries }\end{array}$ & $\begin{array}{l}\text { zero diagonal } \\
\text { entries }\end{array}$ \\
\hline & $\Delta \sim \underline{3}$ & masses for $\mathrm{t} ; \mathrm{b} ; \tau ; \nu_{3}$ & $\begin{array}{c}1-3 \text { and } 2-3 \\
\text { mixing angles }\end{array}$ & $\begin{array}{c}1-3 \text { and } 2-3 \\
\text { mixing angles }\end{array}$ \\
\hline & $\zeta \sim \underline{4}$ & masses for $\mathrm{c}, \mathrm{t} ; \mathrm{s}, \mathrm{b} ; \mu, \tau ; \nu_{2}, \nu_{3}$ & $\begin{array}{l}\text { all entries } \\
\text { non-zero }\end{array}$ & $\begin{array}{l}\text { all entries } \\
\text { non-zero }\end{array}$ \\
\hline
\end{tabular}




\begin{tabular}{|c|c|c|c|c|}
\hline \multirow[t]{4}{*}{$L_{1}+Q_{8}+N_{1}$} & $\phi \sim \underline{1}$ & $\begin{array}{l}\text { all except } \nu \text { 's massive, } \\
\text { many equal masses }\end{array}$ & $U_{K M}=I_{3 \times 3}$ & unphysical \\
\hline & $\chi \sim \underline{2}$ & masses for $\mathrm{c}, \mathrm{t} ; \mathrm{s}, \mathrm{b}$ & Cabibbo angle & unphysical \\
\hline & $\Delta \sim \underline{3}$ & masses for $\mathrm{c}, \mathrm{t} ; \mathrm{s}, \mathrm{b} ; \tau ; \nu_{3}$ & $U_{K M}=I_{3 \times 3}$ & $\begin{array}{l}1-3 \text { and } 2-3 \\
\text { mixing angles }\end{array}$ \\
\hline & $\psi \sim \underline{5}$ & masses for $\mathrm{e}, \mu, \tau$ & unphysical & unphysical \\
\hline \multirow[t]{4}{*}{$L_{1}+Q_{8}+N_{3}$} & $\phi \sim \underline{1}$ & all massive, many equal masses & $U_{K M}=I_{3 \times 3}$ & $U_{e \nu}=I_{3 \times 3}$ \\
\hline & $\chi \sim \underline{2}$ & masses for $\mathrm{c}, \mathrm{t} ; \mathrm{s}, \mathrm{b}$ & Cabibbo angle & unphysical \\
\hline & $\Delta \sim \underline{3}$ & masses for $\mathrm{c}, \mathrm{t} ; \mathrm{s}, \mathrm{b} ; \tau ; \nu_{3}$ & $U_{K M}=I_{3 \times 3}$ & $\begin{array}{l}1-3 \text { and } 2-3 \\
\text { mixing angles }\end{array}$ \\
\hline & $\psi \sim \underline{5}$ & masses for e, $\mu, \tau ; 3 \nu$ 's & unphysical & $\begin{array}{l}1-3 \text { and } 2-3 \\
\text { mixing angles }\end{array}$ \\
\hline \multirow[t]{3}{*}{$L_{1}+Q_{1}+N_{1}$} & $\phi \sim \underline{1}$ & $\begin{array}{c}\text { three equal masses for } \\
\text { quarks and charged leptons }\end{array}$ & $U_{K M}=I_{3 \times 3}$ & unphysical \\
\hline & $\Delta \sim \underline{3}$ & masses for $\mathrm{t} ; \mathrm{b} ; \tau ; \nu_{3}$ & $\begin{array}{c}1-3 \text { and } 2-3 \\
\text { mixing angles }\end{array}$ & $\begin{array}{l}1-3 \text { and } 2-3 \\
\text { mixing angles }\end{array}$ \\
\hline & $\psi \sim \underline{5}$ & all massive except $\nu$ 's & $\begin{array}{l}\text { all entries } \\
\text { non-zero }\end{array}$ & unphysical \\
\hline \multirow[t]{3}{*}{$L_{1}+Q_{1}+N_{3}$} & $\phi \sim \underline{1}$ & three equal masses for each sector & $U_{K M}=I_{3 \times 3}$ & $U_{e \nu}=I_{3 \times 3}$ \\
\hline & $\Delta \sim \underline{3}$ & masses for $\mathrm{t} ; \mathrm{b} ; \tau ; \nu_{3}$ & $\begin{array}{c}1-3 \text { and } 2-3 \\
\text { mixing angles }\end{array}$ & $\begin{array}{l}1-3 \text { and } 2-3 \\
\text { mixing angles }\end{array}$ \\
\hline & $\psi \sim \underline{5}$ & all massive & $\begin{array}{l}\text { all entries } \\
\text { non-zero }\end{array}$ & $\begin{array}{l}\text { all entries } \\
\text { non-zero }\end{array}$ \\
\hline \multirow[t]{3}{*}{$L_{9}+Q_{8}+N_{1}$} & $\phi \sim \underline{1}$ & all massive, some equal & $U_{K M}=I_{3 \times 3}$ & $\begin{array}{l}\text { all entries } \\
\text { non-zero }\end{array}$ \\
\hline & $\chi \sim \underline{2}$ & masses for $\mathrm{c}, \mathrm{t} ; \mathrm{s}, \mathrm{b}$ & Cabibbo angle & unphysical \\
\hline & $\Delta \sim \underline{3}$ & masses for $\mathrm{c}, \mathrm{t} ; \mathrm{s}, \mathrm{b}$ & $U_{K M}=I_{3 \times 3}$ & unphysical \\
\hline \multirow[t]{3}{*}{$L_{9}+Q_{8}+N_{3}$} & $\phi \sim \underline{1}$ & $\begin{array}{c}\text { all massive except } \nu \text { 's, } \\
\text { some equal masses }\end{array}$ & $U_{K M}=I_{3 \times 3}$ & unphysical \\
\hline & $\chi \sim \underline{2}$ & masses for $\mathrm{c}, \mathrm{t} ; \mathrm{s}, \mathrm{b}$ & Cabibbo angle & unphysical \\
\hline & $\Delta \sim \underline{3}$ & masses for $\mathrm{c}, \mathrm{t} ; \mathrm{s}, \mathrm{b} ; \nu_{3}$ & $U_{K M}=I_{3 \times 3}$ & unphysical \\
\hline
\end{tabular}




\begin{tabular}{|c|c|c|c|c|}
\hline \multirow[t]{3}{*}{$L_{9}+Q_{1}+N_{1}$} & $\phi \sim \underline{1}$ & $\begin{array}{l}\text { all massive, } \\
\text { many equal masses }\end{array}$ & $U_{K M}=I_{3 \times 3}$ & $\begin{array}{l}\text { all entries } \\
\text { non-zero }\end{array}$ \\
\hline & $\Delta \sim \underline{3}$ & masses for $\mathrm{t} ; \mathrm{b}$ & $\begin{array}{c}1-3 \text { and } 2-3 \\
\text { mixing angles }\end{array}$ & unphysical \\
\hline & $\psi \sim \underline{5}$ & masses for all quarks & $\begin{array}{l}\text { all entries } \\
\text { non-zero }\end{array}$ & unphysical \\
\hline \multirow[t]{3}{*}{$L_{9}+Q_{1}+N_{3}$} & $\phi \sim \underline{1}$ & $\begin{array}{l}\text { all massive except } \nu \text { 's, } \\
\text { many equal masses }\end{array}$ & $U_{K M}=I_{3 \times 3}$ & unphysical \\
\hline & $\Delta \sim \underline{3}$ & masses for $\mathrm{t} ; \mathrm{b} ; \nu_{3}$ & $\begin{array}{c}1-3 \text { and } 2-3 \\
\text { mixing angles }\end{array}$ & unphysical \\
\hline & $\psi \sim \underline{5}$ & masses for all quarks & $\begin{array}{l}\text { all entries } \\
\text { non-zero }\end{array}$ & unphysical \\
\hline \multirow[t]{3}{*}{$L_{4}+Q_{21}+N_{2}$} & $\phi \sim \underline{1}$ & $\begin{array}{l}\text { all massive, some } \\
\text { equal masses }\end{array}$ & $\begin{array}{l}\text { all entries } \\
\text { non-zero }\end{array}$ & $U_{e \nu}=I_{3 \times 3}$ \\
\hline & $\chi \sim \underline{2}$ & masses for $\mu, \tau ; \nu_{2}, \nu_{3}$ & unphysical & $\begin{array}{c}1-2 \text { or } 1-3 \\
\text { mixing angle }\end{array}$ \\
\hline & $\Delta \sim \underline{3}$ & masses for $\mu, \tau ; \nu_{2}, \nu_{3}$ & unphysical & $U_{e \nu}=I_{3 \times 3}$ \\
\hline \multirow[t]{3}{*}{$L_{1}+Q_{21}+N_{1}$} & $\phi \sim \underline{1}$ & $\begin{array}{c}m_{e}=m_{\mu}=m_{\tau} \\
\text { masses for all quarks }\end{array}$ & $\begin{array}{l}\text { all entries } \\
\text { non-zero }\end{array}$ & unphysical \\
\hline & $\Delta \sim \underline{3}$ & masses for $\tau ; \nu_{3}$ & unphysical & $\begin{array}{l}1-3 \text { and } 2-3 \\
\text { mixing angles }\end{array}$ \\
\hline & $\psi \sim \underline{5}$ & masses for e, $\mu, \tau$ & unphysical & unphysical \\
\hline \multirow[t]{3}{*}{$L_{1}+Q_{21}+N_{3}$} & $\phi \sim 1$ & $\begin{array}{c}\text { all massive, } 3 \text { equal } \\
\text { masses for lepton sectors }\end{array}$ & $\begin{array}{l}\text { all entries } \\
\text { non-zero }\end{array}$ & $U_{e \nu}=I_{3 \times 3}$ \\
\hline & $\Delta \sim \underline{3}$ & masses for $\tau ; \nu_{3}$ & unphysical & $\begin{array}{l}1-3 \text { and } 2-3 \\
\text { mixing angles }\end{array}$ \\
\hline & $\psi \sim \underline{5}$ & masses for all leptons & unphysical & $\begin{array}{l}\text { all entries } \\
\text { non-zero }\end{array}$ \\
\hline
\end{tabular}


TABLE VI. Bounds on Neutrino masses from Accelerator and Double-Beta Decay Measurements [25,39].

\begin{tabular}{l}
\hline \hline Accelerator Limits \\
\hline$\nu_{e} \quad<17 \mathrm{eV}$ \\
\hline$\nu_{\mu} \quad<0.27 \mathrm{MeV}$ \\
\hline$\nu_{\tau} \quad<35 \mathrm{MeV}$ \\
\hline Double-Beta Decay Limit \\
\hline$\left\langle m_{\nu_{e}}\right\rangle=\sum_{i} \xi_{i} U_{e i}^{2} m_{i}<(2 \pm 1) \mathrm{eV}$ \\
\hline \hline
\end{tabular}

